\title{
Obstacle problems for a coupling of quasilinear hyperbolic-parabolic equations
}

\author{
LAURENT LÉVI ${ }^{\dagger}$ \\ Université de Pau et des Pays de l'Adour, \\ Laboratoire de Mathématiques Appliquées, UMR 5142 CNRS, \\ BP 1155, 64013 Pau Cedex, France
}

[Received 29 May 2006 and in revised form 9 February 2007]

\begin{abstract}
We establish the existence and uniqueness of the solution to some inner obstacle problems for a coupling of a multidimensional quasilinear first-order hyperbolic equation set in a region $\Omega_{h}$ with a quasilinear parabolic one set in the complement $\Omega_{p}=\Omega \backslash \Omega_{h}$. The mathematical problem is motivated by physical models for infiltration processes with saturation thresholds.
\end{abstract}

\section{Introduction}

\subsection{Mathematical setting}

Let $\Omega$ be a bounded domain of $\mathbb{R}^{n}, n \geqslant 1$, with a smooth boundary $\Gamma$, and $T$ a positive real number. This paper is devoted to the mathematical analysis of the unilateral and bilateral inner obstacle problems for the coupling of a quasilinear advection-reaction equation of the form

$$
\mathcal{T}_{h}(u)=\partial_{t} u-\sum_{i=1}^{n} \partial_{x_{i}}\left(K(u) B_{i}\right)+g_{h}(t, x, u)=0,
$$

set in a hyperbolic zone $\left.Q_{h}=\right] 0, T\left[\times \Omega_{h}\right.$, with a quasilinear diffusion-advection-reaction equation of the type

$$
\mathcal{T}_{p}(u)=\partial_{t} u-\sum_{i=1}^{n} \partial_{x_{i}}\left(\partial_{x_{i}} \phi(u)+K(u) B_{i}\right)+g_{p}(t, x, u)=0,
$$

set in a parabolic area $\left.Q_{p}=\right] 0, T\left[\times\left(\Omega \backslash \Omega_{h}\right)\right.$, complementary to the former, and for suitable conditions across the interface between the two regions $Q_{p}$ and $Q_{h}$. The geometrical configuration is such that: $\bar{\Omega}=\bar{\Omega}_{h} \cup \bar{\Omega}_{p} ; \Omega_{h}$ and $\Omega_{p}$ are two disjoint bounded domains with Lipschitz boundaries denoted $\Gamma_{l}$, for $l$ in $\{h, p\}$. In addition, the interface $\Gamma_{h p}=\Gamma_{h} \cap \Gamma_{p}$ is Lipschitz and such that $\mathcal{H}^{n-1}\left(\bar{\Gamma}_{h p} \cap \overline{\Gamma_{l} \backslash \Gamma_{h p}}\right)=0$, where for $q$ in $[0, n+1], \mathcal{H}^{q}$ denotes the $q$-dimensional Hausdorff measure.

For a given threshold $\theta$, the (bilateral) obstacle problem for $\mathcal{T}_{h}$ and $\mathcal{T}_{p}$ may be formally written in the free boundary formulation: find a bounded measurable function $u$ on $Q \equiv] 0, T[\times \Omega$ such

\footnotetext{
${ }^{\dagger}$ E-mail: laurent.levi@univ-pau.fr
} 
that

$$
\begin{gathered}
0 \leqslant u \leqslant \theta \quad \text { on } Q, \\
\text { for } i \text { in }\{h, p\}, \quad \mathcal{T}_{i}(u)=0 \quad \text { on } Q_{i} \cap[0<u<\theta], \\
\mathcal{T}_{i}(u) \leqslant 0 \quad \text { on } Q_{i} \cap[0<u=\theta], \quad \mathcal{T}_{i}(u) \geqslant 0 \quad \text { on } Q_{i} \cap[0=u<\theta], \\
u=0 \quad \text { on }] 0, T\left[\times \Gamma, \quad u(0, \cdot)=u_{0} \quad \text { on } \Omega,\right.
\end{gathered}
$$

subject to the transmission conditions (see Remark 3 ) along the interface $\left.\Sigma_{h p}=\right] 0, T\left[\times \Gamma_{h p}\right.$, with $\Gamma_{h p}=\Gamma_{h} \cap \Gamma_{p}$ and $\Gamma_{i}=\partial \Omega_{i}, i \in\{h, p\}$ :

$$
\begin{array}{ll}
u_{\mid Q_{h}}=u_{\mid Q_{p}} & \text { on } \Sigma_{h p} \cap\left[\mathbf{B} \cdot v_{h}>0\right], \\
\nabla \phi(u) \cdot v_{p}=0 & \text { on } \Sigma_{h p} \cap[0<u<\theta],
\end{array}
$$

where $\mathbf{B}=\left(B_{1}, \ldots, B_{n}\right)$ and $v_{i}$ denotes the unit normal outward vector defined $\mathcal{H}^{n}$-a.e. on $\Sigma_{l}$.

REMARK 1 The representation (1)-(3) is also valid for the upper unilateral obstacle problem $(u \leqslant$ $\theta$ ) (resp. for the lower unilateral obstacle problem $(u \geqslant \theta)$ ) by formally replacing the lower bound with " $-\infty$ " (resp. the upper bound with " $+\infty$ "). Observe that in these situations, for $i$ in $\{h, p\}$, $\mathcal{T}_{i}(u)$ are nonpositive (resp. nonnegative) distributions on $Q_{i}$.

This problem arises from several simplified physical models like infiltration processes in a stratified subsoil viewed as two layers with different geological characteristics and such that in the second layer we can neglect the effects of diffusivity. Indeed, when we are interested in the evolution of any effluent $c$ within the flow of substances moving in the subsoil, the first simplified modelling consists in taking into account just one phase saturating the soil, made of two components without any chemical interactions: water and component $c$. We assume that the distribution of temperature $T$ and the pressure field $P$ of the fluid phase are determined, sufficiently smooth functions. Then we invoke P. Bia and M. Combarnous [2] to write the mass conservation law for $c$ and we take into account the existence of some saturation thresholds $\theta_{1, c}(T, P)$ and $\theta_{2, c}(T, P)$; beyond them the appearance of a new phase (liquid or solid) for the same number of components changes the thermodynamical nature of the system, which cannot be described through a simplified balance equation. This way, the relations governing the mass fraction $\omega_{c}$ are formally given by:

$$
\begin{gathered}
\mathcal{E}\left(t, x, \omega_{c}\right)=0 \quad \text { on }\left[\theta_{1}<\omega_{c}<\theta_{2}\right], \\
\mathcal{E}\left(t, x, \omega_{c}\right) \leqslant 0 \quad \text { on }\left[\theta_{1}<\omega_{c}=\theta_{2}\right], \quad \mathcal{E}\left(t, x, \omega_{c}\right) \geqslant 0 \quad \text { on }\left[\theta_{1}=\omega_{c}<\theta_{2}\right], \quad \text { where } \\
\mathcal{E}\left(t, x, \omega_{c}\right)=\rho\left(T, \omega_{c}\right)\left\{\partial_{t} \omega_{c}-\frac{k(x)}{\mu\left(\omega_{c}\right)} \nabla \omega_{c} .\left(\nabla P-\rho\left(T, \omega_{c}\right) \vec{g}\right)\right\} \\
-\operatorname{Div}\left[\mathcal{A}\left(x, \omega_{c}\right) \rho\left(T, \omega_{c}\right) \nabla \omega_{c}\right] .
\end{gathered}
$$

In (7), $k$ denotes the absolute permeability at the point $x, \mu$ the dynamic viscosity of the fluid phase and $\rho\left(T, \omega_{c}\right)$ its density, defined by $\omega_{c}$ at temperature $T$. Lastly, $\vec{g}$ is the gravity acceleration vector. Furthermore, the molecular diffusion-dispersion effects have been taken into account through the tensor $\mathcal{A}(x, \cdot)$. But depending on the geological nature of the subsoil-namely in $\Omega_{h}$-these effects may be neglected in favour of the effluent's transport ones. In this situation the evolution of $\omega_{c}$ is governed by a first-order quasilinear operator. This way, the connection between (7) and (1)-(6) can be achieved for isothermal flows, without gravity effects and for a constant permeability, by 
introducing the new unknown $u_{c}=\int_{0}^{\omega_{c}} \rho(\tau) \mathrm{d} \tau \equiv f\left(\omega_{c}\right)$ and assuming that

$$
K\left(u_{c}\right)=\frac{k}{\mu\left(f^{-1}\left(u_{c}\right)\right)}, \quad \mathbf{B}=\nabla P, \quad g_{i}\left(t, x, u_{c}\right)=K\left(u_{c}\right) \Delta P .
$$

In addition, the "diffusive layer" $\Omega_{p}$ is supposed to be homogeneous and isotropic, so that $\mathcal{A}\left(x, f^{-1}\left(u_{c}\right)\right)$ is reduced to the diagonal matrix $\psi\left(u_{c}\right) \mathcal{I}_{n}$, where $\psi\left(u_{c}\right)$ is a nonlinear function of $u_{c}$.

The paper is organized as follows: in Section 2 we first provide the definition of a weak solution $u$ to (1)-(6) through a global entropy inequality on the whole domain $Q$ and by using the classical Kruzhkov entropy pairs. The uniqueness property is obtained by considering the behaviour of $u$ in the hyperbolic zone and then in the parabolic one where $u$ is characterized by a variational inequality that takes into account entering data from $Q_{h}$. So that on $Q_{p}$ the notion of weak solution and entropy solution to an obstacle problem are equivalent in the sense proposed by K. Kobayasi in [6] for quasilinear degenerate parabolic equations with $L^{1}$-data. In Section 3 the existence theorem uses a viscous regularization and the obstacle condition is relaxed thanks to a penalization procedure. On $Q_{p}$ we pass to the limit with respect to the viscous parameter using classical compactness arguments. However, on $Q_{h}$ we invoke the notion of an entropy process solution, in the spirit of R. Eymard, T. Gallouët \& R. Herbin [4]. It allows us to describe the composite limit $\left(h\left(u_{k}\right)\right)_{k}$ where $h$ is a continuous function on $\mathbb{R}$ and $\left(u_{k}\right)_{k}$ is a bounded sequence in $L^{\infty}(\mathcal{O})$, where $\mathcal{O}$ is an open bounded subset of $\mathbb{R}^{q}(q \geqslant 1)$. Indeed, in this situation, there exists a bounded measurable function

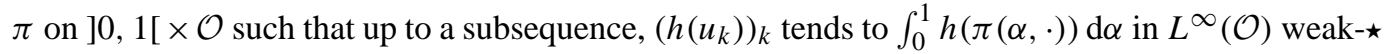
(see Claim 1 for the full statement).

\subsection{Main assumptions on data}

For technical reasons (proofs of Theorem 2 and Proposition 3), we assume in this work that the obstacle $\theta$ is independent of the time variable. So it will be considered as a measurable function on $\Omega$ such that $\theta_{\mid \Omega_{i}}$ belongs to $W^{1, \infty}\left(\Omega_{i}\right), i$ in $\{h, p\}$. In addition $\theta_{\mid \Omega_{p}}$ is an element of $H^{2}\left(\Omega_{p}\right)$. Moreover, $\theta$ is compatible with the boundary condition in the sense that $\theta(\bar{\sigma}) \geqslant 0$ for any $\bar{\sigma}$ in $\partial \Omega$. We set

$$
\forall x \in \Omega, \quad \mathcal{C}_{\theta}(x)=[0, \theta(x)] \quad \text { and } \quad \mathcal{C}_{\theta}^{\infty}=\underset{\bar{\Omega}}{\operatorname{ess} \sup \theta]}
$$

for the bilateral obstacle problem, while

$$
\left.\left.\left.\left.\mathcal{C}_{\theta}(x)=\right]-\infty, \theta(x)\right] \quad \text { and } \quad \mathcal{C}_{\theta}^{\infty}=\right]-\infty, \underset{\bar{\Omega}}{\operatorname{ess} \sup \theta}\right]
$$

for the (upper) unilateral obstacle problem, the reasoning for lower and upper unilateral obstacle problems being similar.

The vector field $\mathbf{B}$ is in $W^{2, \infty}(Q)^{n}$. In particular, $B_{i}$ and $\partial_{x_{j}} B_{i}$ are continuous on the whole $\Omega$ with respect to the space variable. Moreover,

$$
\Sigma_{h p} \subset\left\{\sigma \in \Sigma_{h}: \mathbf{B}(\sigma) . v_{h} \leqslant 0\right\} .
$$

The initial data $u_{0}$ belongs to $L^{\infty}(\Omega) \cap H_{0}^{1}(\Omega)$. In addition, $u_{0} \in \mathcal{C}_{\theta}(x)$ for a.e. $x$ in $\Omega$. Moreover, for $i$ in $\{h, p\}$, the reaction term $g_{i}$ is in $W^{1, \infty}(] 0, T\left[\times \Omega_{i} \times \mathcal{C}_{\theta}^{\infty}\right)$ and we set

$$
M_{g_{i}}=\operatorname{ess~sup}_{(t, x, u) \in] 0, T\left[\times \Omega_{i} \times \mathcal{C}_{\theta}^{\infty}\right.}\left|\partial_{u} g_{i}(t, x, u)\right| .
$$


The transport term $K$ is Lipschitz continuous on $\mathcal{C}_{\theta}^{\infty}$ with a constant $\mathcal{K}_{K}$. Furthermore, $K$ is nondecreasing. Thus we may define the nonnegative and nondecreasing function

$$
M_{1}: t \in[0, T] \mapsto M_{1}(t)=\underset{\Omega}{\operatorname{ess} \sup } \theta e^{\mathcal{N} t}+\frac{N_{3}}{\mathcal{N}}\left(e^{\mathcal{N} t}-1\right)
$$

where

$$
\begin{aligned}
\mathcal{N} & =\sum_{i \in\{h, p\}} M_{g_{i}}+\mathcal{K}_{K}\|\operatorname{div} \mathbf{B}\|_{L^{\infty}(Q)}, \\
N_{3} & =\sum_{i \in\{h, p\}}{\operatorname{ess} \sup _{0, T\left[\times \Omega_{i}\right.}}_{g_{i}}(t, x, 0)^{-}+\underset{] 0, T[\times \Omega}{\operatorname{ess} \sup }(K(0) \operatorname{div} \mathbf{B})^{-} .
\end{aligned}
$$

We also introduce the nonpositive and nonincreasing function

$$
M_{2}: t \in[0, T] \mapsto M_{2}(t)=\min \left(0, \underset{\Omega}{\operatorname{essinf}} u_{0}\right) e^{\mathcal{N} t}+\frac{N_{4}}{\mathcal{N}}\left(e^{\mathcal{N} t}-1\right),
$$

with

$$
N_{4}=-\sum_{i \in\{h, p\}]} \operatorname{ess} \sup _{0, T\left[\times \Omega_{i}\right.} g_{i}(t, x, 0)^{+}-\underset{] 0, T[\times \Omega}{\operatorname{ess} \sup (K(0) \operatorname{div} \mathbf{B})^{+} .}
$$

From now, to unify the presentation with the bilateral obstacle problem we set, for the unilateral obstacle problem,

$$
\mathcal{C}_{\theta}^{\infty}=\left[M_{2}(T), \underset{\bar{\Omega}}{\operatorname{ess} \sup \theta]}\right.
$$

Lastly, $\phi$ is a nondecreasing function in $W^{1, \infty}\left(\mathcal{C}_{\theta}^{\infty}\right)$ with $\phi(0)=0, \phi^{\prime}$ is Lipschitz continuous on $\mathcal{C}_{\theta}^{\infty}$ and

$$
\phi^{-1} \text { exists and is continuous on } \phi\left(\mathcal{C}_{\theta}^{\infty}\right) \text {. }
$$

We point out that $(11)$ is in particular satisfied when $\mathcal{L}\left(\left\{x \in \mathcal{C}_{\theta}^{\infty}: \phi^{\prime}(x)=0\right\}\right)=0$, where $\mathcal{L}$ is the Lebesgue measure on $\mathbb{R}$.

REMARK 2 The monotonicity of $K$ and (8) show that the interface $\Sigma_{h p}$ is included in the set of outward characteristics for the first-order operator in the hyperbolic domain. So in the transmission zone, (5) is useless since the data leave the hyperbolic domain. This essential property will guide us for the statement of uniqueness; we will first consider the behaviour of a solution in the hyperbolic area and then in the parabolic one.

\subsection{Notations and function spaces}

In the following, $\sigma$ (resp. $\bar{\sigma}$ ) is a variable in $\Sigma_{i}$ (resp. $\left.\Gamma_{i}\right), i \in\{h, h p, p\}$. Thus, $\sigma=(t, \bar{\sigma})$ for any $t$ in $[0, T]$.

We need to consider the Hilbert space

$$
V=\left\{v \in H^{1}\left(\Omega_{p}\right): v=0 \text { a.e. on } \Gamma_{p} \backslash \Gamma_{h p}\right\}
$$

with the norm $\|v\|_{V}=\|\nabla v\|_{L^{2}\left(\Omega_{p}\right)^{n}}$, equivalent to the classical $H^{1}(\Omega)$-norm. We denote by $\langle\langle\cdot, \cdot\rangle\rangle$ the pairing between $V$ and $V^{\prime}$, and by $\langle\cdot, \cdot\rangle$ the pairing between $H_{0}^{1}(\Omega)$ and $H^{-1}(\Omega)$. Furthermore, for $X$ and $Y$ two Hilbert spaces, we will make use of the Hilbert space

$$
W(0, T ; X ; Y) \equiv\left\{v \in L^{2}(0, T ; X): \partial_{t} v \in L^{2}(0, T ; Y)\right\},
$$


equipped with the norm $\|v\|_{W(0, T ; X ; Y)}=\left(\left\|\partial_{t} v\right\|_{L^{2}(0, T ; Y)}^{2}+\|v\|_{L^{2}(0, T ; X)}^{2}\right)^{1 / 2}$. In what follows, $X$ will be mainly taken to be $H_{0}^{1}(\Omega)$ or $V$, and $Y$ to be $H^{-1}(\Omega)$ or $V^{\prime}$ respectively.

The function $\operatorname{sgn}_{\mu}$ denotes the Lipschitzian and bounded approximation of the function sgn given for any positive $\mu$ and any nonnegative real $x$ by

$$
\operatorname{sgn}_{\mu}(x)=\min (x / \mu, 1) \quad \text { and } \quad \operatorname{sgn}_{\mu}(-x)=-\operatorname{sgn}_{\mu}(x) .
$$

Lastly, to simplify the writing, we set for $i$ in $\{h, p\}$ :

$$
\begin{aligned}
G_{i}(u, v) & =g_{i}(t, x, u)-\operatorname{div}(K(v) \mathbf{B}), \\
L_{i}(u, v, w) & =-|u-v| \partial_{t} w-|K(u)-K(v)| \mathbf{B} . \nabla w-\operatorname{sgn}(u-v) G_{i}(u, v) w,
\end{aligned}
$$

and with $\mathbb{I}_{\Omega_{i}}(x)=1$ if $x \in \Omega_{i}, \mathbb{I}_{\Omega_{i}}(x)=0$ otherwise,

$$
\begin{aligned}
L(u, v, w) & =L_{p}(u, v, w) \mathbb{I}_{\Omega_{p}}(x)+L_{h}(u, v, w) \mathbb{I}_{\Omega_{h}}(x), \\
g(t, x, u) & =g_{p}(t, x, u) \mathbb{I}_{\Omega_{p}}(x)+g_{h}(t, x, u) \mathbb{I}_{\Omega_{h}}(x), \\
\mathcal{F}(u, v, w) & =\frac{1}{2}\{|K(u)-K(v)|-|K(w)-K(v)|+|K(u)-K(w)|\} .
\end{aligned}
$$

\section{Statement of uniqueness}

We give the definition of a weak solution to (1)-(6) by first keeping in mind that it has to involve an entropy criterion on $Q_{h}$ and secondly by taking into account the obstacle condition for $u$. That is why, by noting that (1)-(6) can be viewed as an obstacle problem for a quasilinear parabolic evolution equation that strongly degenerates on a fixed subdomain, we make use of related work ([1], [8]) to propose a weak formulation through a global entropy inequality on the whole $Q$, the latter giving rise to a variational inequality on the parabolic domain, and to an entropy inequality on the hyperbolic one so as to ensure uniqueness.

\subsection{Definition}

So we now formulate

Definition 1 A measurable function $u$ is a weak solution to (1p-(6) if

$$
\begin{aligned}
& \text { for a.e. } t \text { in }] 0, T\left[, \quad u(t, \cdot) \in \mathcal{C}_{\theta} \quad \text { a.e. in } \Omega, \quad \phi(u) \in L^{2}(0, T ; V),\right. \\
& \begin{array}{r}
\forall \zeta \in \mathcal{D}(Q), \zeta \geqslant 0, \\
\int_{Q} L(u, \kappa, \zeta) \mathrm{d} x \mathrm{~d} t-\int_{Q_{p}} \nabla|\phi(u)-\phi(\kappa)| . \nabla \zeta \mathrm{d} x \mathrm{~d} t+\int_{Q_{p}} \Delta \phi(\kappa) \operatorname{sgn}(u-\kappa) \zeta \mathrm{d} x \mathrm{~d} t \\
\quad+\int_{\Sigma_{h p}} \nabla \phi(\kappa) . \nu_{h} \operatorname{sgn}(\phi(u)-\phi(\kappa)) \zeta \mathrm{d} \mathcal{H}^{n} \geqslant 0,
\end{array}
\end{aligned}
$$

where $\kappa=k \theta, k \in[0,1]$ for the bilateral obstacle problem and $\kappa=k+\theta, k \in\left[M_{2}(T)-\right.$ $\left.\operatorname{ess}_{\sup } \bar{\Omega} \theta, 0\right]$ for the unilateral problem, and 
$\forall \zeta \in L^{1}\left(\Sigma_{h} \backslash \Sigma_{h p}\right), \zeta \geqslant 0$

$$
\begin{gathered}
\underset{\tau \rightarrow 0^{-}}{\operatorname{ess} \lim } \int_{\Sigma_{h} \backslash \Sigma_{h p}} \mathcal{F}\left(u\left(\sigma+\tau \nu_{h}\right), 0, \kappa(\bar{\sigma})\right) \mathbf{B}(\sigma) . v_{h} \zeta \mathrm{d} \mathcal{H}^{n} \leqslant 0, \\
\underset{t \rightarrow 0^{+}}{\operatorname{ess} \lim } \int_{\Omega}\left|u(t, x)-u_{0}(x)\right| \mathrm{d} x=0 .
\end{gathered}
$$

REMARK 3 (i) When $\theta$ is nonnegative on $Q$, the formulation for the (upper) unilateral obstacle problem is a special case of 13 for the bilateral obstacle problem by considering $k \leqslant 1$ only.

(ii) The link between (2), 3), (6) and (13) can be achieved through two inequalities resulting from (13); that will be useful in what follows. In (13), we take $\kappa(x)=\theta(x)$, which means $k=1$ in the case of a bilateral obstacle condition and $k=0$ in the case of a unilateral one. It follows that (with $\left.\mathcal{T}=\mathcal{T}_{h} \mathbb{I}_{\Omega_{h}}+\mathcal{T}_{p} \mathbb{I}_{\Omega_{p}}\right)$

$$
\begin{aligned}
\int_{Q} u \partial_{t} \zeta \mathrm{d} x \mathrm{~d} t \leqslant & \int_{Q_{p}}(\nabla \phi(u)+K(u) \mathbf{B}) \cdot \nabla \zeta \mathrm{d} x \mathrm{~d} t+\int_{Q} g(t, x, u) \zeta \mathrm{d} x \mathrm{~d} t \\
& +\int_{\Sigma_{h p}} \nabla \phi(\theta) \cdot v_{h}(1+\operatorname{sgn}(\phi(u)-\phi(\theta))) \zeta \mathrm{d} \mathcal{H}^{n} \\
& -\int_{Q}(1+\operatorname{sgn}(u-\theta)) \mathcal{T}(\theta) \zeta \mathrm{d} x \mathrm{~d} t .
\end{aligned}
$$

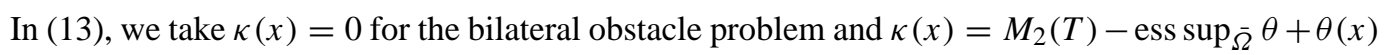
for the unilateral one (so that $u-\kappa \geqslant 0$ a.e.). One has

$$
\begin{aligned}
\int_{Q} u \partial_{t} \zeta \mathrm{d} x \mathrm{~d} t \geqslant & \int_{Q_{p}}(\nabla \phi(u)+K(u) \mathbf{B}) \cdot \nabla \zeta \mathrm{d} x \mathrm{~d} t+\int_{Q} g(t, x, u) \zeta \mathrm{d} x \mathrm{~d} t \\
& +\int_{\Sigma_{h p}} \nabla \phi(\kappa) \cdot v_{h}(1-\operatorname{sgn}(\phi(u)-\phi(\kappa))) \zeta \mathrm{d} \mathcal{H}^{n} \\
& -\int_{Q}(1-\operatorname{sgn}(u-\kappa)) \mathcal{T}(\kappa) \zeta \mathrm{d} x \mathrm{~d} t .
\end{aligned}
$$

Let $\mathbf{V}=\left(u,-\mathbb{I}_{\Omega_{p}} \nabla \phi(u)-K(u) \mathbf{B}\right)$ in $L^{2}(Q)^{n+1}$. For any $\zeta$ in $\mathcal{D}\left(Q_{i}\right), i$ in $\{h, p\}$, we take $\zeta^{+}$ and $\zeta^{-}$as test functions in 16 and 17 . By writing $\zeta=\zeta^{+}-\zeta^{-}$, we deduce the existence of a constant $C$ such that

$$
\left|\int_{Q_{i}} \mathbf{V} \cdot\left(\partial_{t} \zeta, \nabla \zeta\right) \mathrm{d} x \mathrm{~d} t\right| \leqslant C\|\zeta\|_{L^{2}\left(Q_{i}\right)}
$$

That means $\mathbf{V}_{\mid Q_{i}} \in H_{\operatorname{div}_{(t, x)}}\left(Q_{i}\right)=\left\{\mathbf{v} \in L^{2}\left(Q_{i}\right)^{n+1}: \operatorname{div}_{(t, x)} \mathbf{v} \in L^{2}\left(Q_{i}\right)\right\}$. We deduce that $\mathbf{V}_{\mid Q_{i}} \cdot v_{i} \in H_{00}^{-1 / 2}\left(\Sigma_{h p}\right)$, the topological dual of

$$
H_{00}^{1 / 2}\left(\Sigma_{h p}\right)=\left\{v \in L^{2}\left(\Sigma_{h p}\right): \exists w \in H_{0}^{1}(Q), v=w_{\mid \Sigma_{h p}}\right\} .
$$

In addition, we derive from (16) and (17) that a.e. on $Q_{i}$,

$$
\begin{aligned}
& -\operatorname{div}_{(t, x)} \mathbf{V}_{\mid Q_{i}} \leqslant g_{i}(t, x, u)-(1+\operatorname{sgn}(u-\theta)) \mathcal{T}_{i}(\theta), \\
& -\operatorname{div}_{(t, x)} \mathbf{V}_{\mid Q_{i}} \geqslant g_{i}(t, x, u)-(1-\operatorname{sgn}(u-\kappa)) \mathcal{T}_{i}(\kappa) .
\end{aligned}
$$


We multiply each inequality by $\zeta$ in $\mathcal{D}(Q), \zeta \geqslant 0$, and add up with respect to $i$. By denoting $\lfloor\cdot, \cdot \cdot$ the pairing between $H_{00}^{1 / 2}\left(\Sigma_{h p}\right)$ and $H_{00}^{-1 / 2}\left(\Sigma_{h p}\right)$ we obtain

$\int_{Q} \mathbf{V} .\left(\partial_{t} \zeta, \nabla \zeta\right) \mathrm{d} x \mathrm{~d} t-\left\lfloor\mathbf{V}_{\mid Q_{h}} \cdot v_{h}+\mathbf{V}_{\mid Q_{p}} \cdot v_{p}, \zeta\right\rfloor \leqslant \int_{Q}(g(t, x, u)-(1+\operatorname{sgn}(u-\theta)) \mathcal{T}(\theta)) \zeta \mathrm{d} x \mathrm{~d} t$ and

$\int_{Q} \mathbf{V} .\left(\partial_{t} \zeta, \nabla \zeta\right) \mathrm{d} x \mathrm{~d} t-\left\lfloor\mathbf{V}_{\mid Q_{h}} \cdot v_{h}+\mathbf{V}_{\mid Q_{p}} \cdot v_{p}, \zeta\right\rfloor \geqslant \int_{Q}(g(t, x, u)-(1-\operatorname{sgn}(u-\kappa)) \mathcal{T}(\kappa)) \zeta \mathrm{d} x \mathrm{~d} t$.

Now what follows is formal. We are interested in the bilateral obstacle problem (the reasoning for the unilateral one being similar) and assume that $[0<u<\theta]$ is an open $\mathcal{H}^{n}$-measurable subset of $Q$. We assume that $\zeta$ in (16) and (17) has compact support in $Q_{i} \cap[0<u<\theta]$. As $(1+\operatorname{sgn}(u-\theta)) \zeta=$ $(1-\operatorname{sgn}(u-\kappa)) \zeta=0$ a.e. and $(1+\operatorname{sgn}(\phi(u)-\phi(\theta))) \zeta=(1-\operatorname{sgn}(\phi(u)-\phi(\kappa))) \zeta=0 \mathcal{H}^{n}$-a.e., we deduce that for $i$ in $\{h, p\}$,

$$
\operatorname{div}_{(t, x)}\left(\mathbf{V}_{\mid Q_{i}}\right)=0 \quad \text { on } Q_{i} \cap[0<u<\theta],
$$

that is, (2). Then, for $\zeta$ with compact support in $Q \cap[0<u<\theta]$, by comparing (16) and (17) with the above inequality, we get

$$
\left\lfloor\mathbf{V}_{\mid Q_{h}} \cdot v_{h}+\mathbf{V}_{\mid Q_{p}} \cdot v_{p}, \zeta\right\rfloor=0
$$

which is (6) in a certain sense. Furthermore, if we take $\zeta$ with support in $Q_{i} \cap[0<u=\theta]$ and in $Q_{i} \cap[0=u<\theta]$-if it is meaningful—we find $(3)$. Moreover, for any nonnegative $\zeta$ with compact support in $Q \cap[0<u=\theta]$,

$$
\left\lfloor\mathbf{V}_{\mid Q_{h}} \cdot v_{h}+\mathbf{V}_{\mid Q_{p}} \cdot v_{p}, \zeta\right\rfloor=\int_{\Sigma_{h p}} \nabla \phi(\theta) \cdot v_{h} \zeta \mathrm{d} \mathcal{H}^{n}
$$

and

$$
\left\lfloor\mathbf{V}_{\mid Q_{h}} \cdot v_{h}+\mathbf{V}_{\mid Q_{p}} \cdot v_{p}, \zeta\right\rfloor \geqslant \int_{Q} \mathcal{T}(\theta) \zeta \mathrm{d} x \mathrm{~d} t
$$

Thus,

$$
\nabla \phi(\theta) . v_{h} \geqslant \mathcal{T}(\theta) \quad \mathcal{H}^{n} \text {-a.e. on } \Sigma_{h p} \cap[0<u=\theta] .
$$

On $\Sigma_{h p} \cap[0=u<\theta]$, since $K(0)=\phi(0)=0$, for any nonnegative $\zeta$ with compact support in $Q \cap[0=u<\theta]$,

$$
\left\lfloor\mathbf{V}_{\mid Q_{h}} \cdot v_{h}+\mathbf{V}_{\mid Q_{p}} \cdot v_{p}, \zeta\right\rfloor=0 \leqslant \int_{Q} g(t, x, 0) \zeta \mathrm{d} x \mathrm{~d} t .
$$

\subsection{Study in the hyperbolic zone}

We derive from (13) and (14) an entropy inequality on the hyperbolic domain that will be the starting point to establish a Lipschitzian time-dependence in $L^{1}\left(\Omega_{h}\right)$ of a weak solution to (1)-(6) with respect to the corresponding initial data. To do so we need a lemma proved as in [1]: 
LEMMA 1 Let $u$ be a bounded measurable function on $Q$ satisfying (13) and (14). Then for any $\kappa$ as in Definition 1 and any $\varphi$ in $\mathcal{D}(] 0, T\left[\times \mathbb{R}^{n}\right), \varphi \geqslant 0$,

$$
\begin{aligned}
-\int_{Q_{h}} L_{h}(u, \kappa, \zeta) \mathrm{d} x \mathrm{~d} t \leqslant & -\underset{\tau \rightarrow 0^{-}}{\operatorname{ess} \lim } \int_{\Sigma_{h} \backslash \Sigma_{h p}}\left|K\left(u\left(\sigma+\tau v_{h}\right)\right)\right| \mathbf{B}(\sigma) \cdot v_{h} \varphi(\sigma) \mathrm{d} \mathcal{H}^{n} \\
& +\int_{\Sigma_{h} \backslash \Sigma_{h p}}|K(\kappa)(\sigma)| \mathbf{B}(\sigma) \cdot v_{h} \varphi(\sigma) \mathrm{d} \mathcal{H}^{n} .
\end{aligned}
$$

In order to use the method of doubling variables, we now need a technical result already pointed out in [11, proof of Proposition 1]. From [18] we find that for any open subset $\Sigma_{\text {loc }}$ of $\Sigma_{h}$ and $\kappa$ as in Definition 1 ,

$$
\underset{\tau \rightarrow 0^{-}}{\operatorname{ess} \lim } \int_{\Sigma_{\mathrm{loc}}}\left|K\left(u\left(\sigma+\tau \nu_{h}\right)\right)-K(\kappa(\sigma))\right| \mathbf{B}(\sigma) \cdot v_{h} \beta(\sigma) \mathrm{d} \mathcal{H}^{n} \text { exists }
$$

and there exists $\gamma \in L^{\infty}\left(\Sigma_{\text {loc }}\right)$ such that

$$
\underset{\tau \rightarrow 0^{-}}{\operatorname{ess} \lim } \int_{\Sigma_{\mathrm{loc}}} K\left(u\left(\sigma+\tau \nu_{h}\right)\right) \mathbf{B}(\sigma) \cdot \nu_{h} \beta(\sigma) \mathrm{d} \mathcal{H}^{n}=\int_{\Sigma_{\mathrm{loc}}} \gamma_{\kappa}(\sigma) \beta(\sigma) \mathrm{d} \mathcal{H}^{n}
$$

for any $\beta$ in $L^{1}\left(\Sigma_{\text {loc }}\right)$. In the following, 19 and will be used with $\Sigma_{\text {loc }}=\Sigma_{h p}$ or $\Sigma_{\text {loc }}=$ $\Sigma_{h} \backslash \Sigma_{h p}$. We define the sequence $\left(\mathcal{W}_{\delta}\right)_{\delta>0}$ of functions on $\mathbb{R}^{n+1}$,

$$
\forall \delta>0, \forall p=(t, x) \in \mathbb{R}^{n+1}, \quad \mathcal{W}_{\delta}(p)=\rho_{\delta}(t) \prod_{i=1}^{n} \rho_{\delta}\left(x_{i}\right),
$$

where $\left(\rho_{\delta}\right)_{\delta>0}$ is a standard sequence of mollifiers on $\mathbb{R}$. We apply on $\Sigma_{h} \backslash \Sigma_{h p}$ the proof developed in [12, Lemma 3.3] based on properties of mollifiers on the whole boundary to state:

LEMMA 2 Let $u$ be a bounded measurable function on $Q_{h}$ such that 19 holds. Then for any continuous function $\varphi$ on $Q_{h} \cup \Sigma_{h}$,

$$
\begin{aligned}
\lim _{\delta \rightarrow 0^{+}} \int_{Q_{h}} \int_{\Sigma_{h} \backslash \Sigma_{h p}}|K(u(p))| \mathbf{B}(\tilde{\sigma}) . & v_{h} \varphi\left(\frac{\tilde{\sigma}+p}{2}\right) \mathcal{W}_{\delta}(\tilde{\sigma}-p) \mathrm{d} \mathcal{H}_{\tilde{\sigma}}^{n} \mathrm{~d} p \\
= & \frac{1}{2} \underset{\tau \rightarrow 0^{-}}{\operatorname{ess} \lim } \int_{\Sigma_{h} \backslash \Sigma_{h p}}\left|K\left(u\left(\sigma+\tau v_{h}\right)\right)\right| \mathbf{B}(\sigma) v_{h} \varphi(\sigma) \mathrm{d} \mathcal{H}^{n}
\end{aligned}
$$

and

$$
\begin{aligned}
& \lim _{\delta \rightarrow 0^{+}} \int_{Q_{h}} \underset{\tau \rightarrow 0^{-}}{\operatorname{ess} \lim } \int_{\Sigma_{h} \backslash \Sigma_{h p}}\left|K\left(u\left(\sigma+\tau \nu_{h}\right)\right)\right| \mathbf{B}(\sigma) \cdot v_{h} \varphi\left(\frac{\sigma+\tilde{p}}{2}\right) \mathcal{W}_{\delta}(\sigma-\tilde{p}) \mathrm{d} \mathcal{H}_{\sigma}^{n} \mathrm{~d} \tilde{p} \\
& =\frac{1}{2} \underset{\tau \rightarrow 0^{-}}{\operatorname{ess} \lim } \int_{\Sigma_{h} \backslash \Sigma_{h p}}\left|K\left(u\left(\sigma+\tau v_{h}\right)\right)\right| \mathbf{B}(\sigma) \cdot \nu_{h} \varphi(\sigma) \mathrm{d} \mathcal{H}^{n} .
\end{aligned}
$$

From Lemmas 1 and 2 we derive:

THEOREM 1 Let $u_{1}$ and $u_{2}$ be two bounded measurable functions on $Q_{h}$, with $u_{1}(t, \cdot)$ and $u_{2}(t, \cdot)$ in $\mathcal{C}_{\theta}$ a.e. on $Q_{h}$ for a.e. $t$ in ]0,T [, satisfying [18) and (15) respectively for initial data $u_{0,1}$ and $u_{0,2}$. Then

for a.e. $t$ in $] 0, T\left[, \quad \int_{\Omega_{h}}\left|u_{1}(t, \cdot)-u_{2}(t, \cdot)\right| \mathrm{d} x \leqslant e^{M_{g_{h}} t} \int_{\Omega_{h}}\left|u_{0,1}-u_{0,2}\right| \mathrm{d} x\right.$. 
Proof. We choose in (18), for $u_{1}$ written in variables $p=(t, x)$,

$$
\kappa(x)=u_{2}(\tilde{t}, \tilde{x})-\theta(\tilde{x})+\theta(x)
$$

in the case of an (upper) unilateral constraint, while

$$
\kappa(x)= \begin{cases}\frac{u_{2}(\tilde{t}, \tilde{x})}{\theta(\tilde{x})} \theta(x) & \text { if } \theta(\tilde{x}) \neq 0, \\ 0 & \text { else, }\end{cases}
$$

for a bilateral obstacle condition, and similarly in $\sqrt{18}$ for $u_{2}$ written in variables $\tilde{p}=(\tilde{t}, \tilde{x})$. Furthermore in 18 for $u_{1}$,

$$
\varphi(p)=\zeta\left(\frac{p+\tilde{p}}{2}\right) \mathcal{W}_{\delta}(p-\tilde{p}),
$$

where $\delta$ is positive and large enough, and $\zeta \in \mathcal{D}(] 0, T\left[\times \mathbb{R}^{n}\right), \zeta \geqslant 0$; and similarly in (18) for $u_{2}$. We integrate over $Q_{h}$ in the $\tilde{p}$ variables for $u_{1}$ and in the $p$ variables for $u_{2}$. We add up. Through techniques developed in [8] we let $\delta \rightarrow 0$ on the left-hand side. Then the right-hand side goes to 0 , thanks to Lemma 2 for $u_{1}$ and $u_{2}$. It follows that

$$
\begin{aligned}
-\int_{Q_{h}}\left\{\left|u_{1}-u_{2}\right| \partial_{t} \zeta-\mid K\left(u_{1}\right)\right. & \left.-K\left(u_{2}\right) \mid \mathbf{B} \cdot \nabla \zeta\right\} \mathrm{d} x \mathrm{~d} t \\
& \leqslant-\int_{Q_{h}} \operatorname{sgn}\left(u_{1}-u_{2}\right)\left(g_{h}\left(t, x, u_{1}\right)-g_{h}\left(t, x, u_{2}\right)\right) \zeta \mathrm{d} x \mathrm{~d} t .
\end{aligned}
$$

For $\zeta \equiv \alpha \psi$ where $\alpha \in \mathcal{D}(] 0, T[), \alpha \geqslant 0$ and $\psi \in \mathcal{D}\left(\mathbb{R}^{n}\right), \psi \geqslant 0, \psi \equiv 1$ on $Q_{h}$, the Lipschitz condition for $g_{h}$ provides

$$
-\int_{Q_{h}}\left|u_{1}-u_{2}\right| \alpha^{\prime}(t) \mathrm{d} x \mathrm{~d} t \leqslant M_{g_{h}}^{\prime} \int_{Q_{h}}\left|u_{1}-u_{2}\right| \alpha(t) \mathrm{d} x \mathrm{~d} t .
$$

When $\alpha$ is an element of a sequence approximating $\mathbb{I}_{[0, t]}$, for $t$ outside a set of measure zero, the desired inequality is obtained thanks to the initial condition $[15)$ for $u_{1}$ and $u_{2}$ and to Gronwall's lemma.

\subsection{Study in the parabolic zone}

We now consider the behaviour of a weak solution $u$ to (1)-(6) on the parabolic domain. With this in view, we characterize $u$ on $Q_{p}$ through a strong variational inequality (in the sense of J.-L. Lions [10]) including the contribution of entering data from the hyperbolic zone. Indeed:

Proposition 1 Let $u$ be a bounded measurable function on $Q$ such that $\nabla \phi(u) \in L^{2}\left(Q_{p}\right)^{n}$ and (13) holds. Then $\partial_{t} u \in L^{2}\left(0, T ; V^{\prime}\right)$. Furthermore, for any $v$ in $L^{2}(0, T ; V)$ such that for a.e. $t$ in ] $0, T\left[, \phi^{-1}(v(t, \cdot)) \in \mathcal{C}_{\theta}\right.$ a.e. on $\Omega_{p}$,

$$
\begin{aligned}
& \int_{0}^{T}\left\langle\left\langle\partial_{t} u, v-\phi(u)\right\rangle\right\rangle \mathrm{d} t+\int_{Q_{p}}(\nabla \phi(u)+K(u) \mathbf{B}) \cdot \nabla(v-\phi(u)) \mathrm{d} x \mathrm{~d} t+\int_{Q_{p}} g_{p}(t, x, u)(v-\phi(u)) \mathrm{d} x \mathrm{~d} t \\
&+\underset{\tau \rightarrow 0^{-}}{\operatorname{ess} \lim } \int_{\Sigma_{h p}} K\left(u\left(\sigma+\tau v_{h}\right)\right) \mathbf{B}(\sigma) \cdot v_{h}(v-\phi(u)) \mathrm{d} \mathcal{H}^{n} \geqslant 0 .
\end{aligned}
$$


Proof. Thanks to a density argument, (16) and (17) still hold for any nonnegative $\zeta$ in $\mathcal{D}\left(0, T ; H_{0}^{1}(\Omega)\right)$. Now let $\varphi \in \mathcal{D}(0, T ; V)$. Let $\hat{\varphi}$ be an extension of $\varphi$ to $\mathcal{D}\left(0, T ; H_{0}^{1}(\Omega)\right)$ and take $\zeta=\hat{\varphi} \xi_{\varrho}$ in 16 and 17 , where $\xi_{\varrho} \in W^{1, \infty}(\Omega), 0 \leqslant \xi_{\varrho} \leqslant 1$, and for any positive $\varrho$,

$$
\xi_{\varrho}(x)= \begin{cases}1 & \text { if } x \in \bar{\Omega}_{p}, \\ 0 & \text { if } x \in \Omega_{h}, \operatorname{dist}\left(x, \Gamma_{h p}\right) \geqslant \varrho,\left\|\nabla \xi_{\varrho}\right\|_{\infty} \leqslant C / \varrho .\end{cases}
$$

To pass to the limit as $\varrho \rightarrow 0^{+}$, we claim that due to 19 (see [1]),

$$
\lim _{\varrho \rightarrow 0^{+}} \int_{Q_{h}} K(u) \hat{\varphi} \mathbf{B} \cdot \nabla \xi_{\varrho} \mathrm{d} x \mathrm{~d} t=\underset{\tau \rightarrow 0^{-}}{\operatorname{ess}} \int_{\Sigma_{h p}} K\left(u\left(\sigma+\tau v_{h}\right)\right) \varphi(\sigma) \mathbf{B} \cdot v_{h} \mathrm{~d} \mathcal{H}^{n} .
$$

This way, for any $\varphi$ in $\mathcal{D}(0, T ; V), \varphi \geqslant 0$,

$$
\begin{aligned}
\int_{Q_{p}} u \partial_{t} \varphi \mathrm{d} x \mathrm{~d} t \leqslant & \int_{Q_{p}}(\nabla \phi(u)+K(u) \mathbf{B}) \cdot \nabla \varphi \mathrm{d} x \mathrm{~d} t+\int_{Q_{p}} g_{p}(t, x, u) \varphi \mathrm{d} x \mathrm{~d} t \\
& +\int_{\Sigma_{h p}} \nabla \phi(\theta) \cdot v_{h}(1+\operatorname{sgn}(u-\theta)) \varphi \mathrm{d} \mathcal{H}^{n} \\
& -\int_{Q_{p}}(1+\operatorname{sgn}(u-\theta)) \mathcal{T}_{p}(\theta) \varphi \mathrm{d} x \mathrm{~d} t \\
& +\underset{\tau \rightarrow 0^{-}}{\operatorname{ess} \lim } \int_{\Sigma_{h p}} K\left(u\left(\sigma+\tau v_{h}\right)\right) \varphi(\sigma) \mathbf{B} \cdot v_{h} \mathrm{~d} \mathcal{H}^{n}
\end{aligned}
$$

and

$$
\begin{aligned}
\int_{Q_{p}} u \partial_{t} \varphi \mathrm{d} x \mathrm{~d} t \geqslant & \int_{Q_{p}}(\nabla \phi(u)+K(u) \mathbf{B}) \cdot \nabla \varphi \mathrm{d} x \mathrm{~d} t+\int_{Q_{p}} g_{p}(t, x, u) \varphi \mathrm{d} x \mathrm{~d} t \\
& +\int_{\Sigma_{h p}} \nabla \phi(\kappa) \cdot v_{h}\left(1-\operatorname{sgn}\left(u_{\epsilon}-\kappa\right)\right) \varphi \mathrm{d} \mathcal{H}^{n} \\
& -\int_{Q_{p}}(1-\operatorname{sgn}(u-\kappa)) \mathcal{T}_{p}(\kappa) \varphi \mathrm{d} x \mathrm{~d} t \\
& +\underset{\tau \rightarrow 0^{-}}{\operatorname{ess} \lim } \int_{\Sigma_{h p}} K\left(u\left(\sigma+\tau v_{h}\right)\right) \varphi(\sigma) \mathbf{B} \cdot v_{h} \mathrm{~d} \mathcal{H}^{n} .
\end{aligned}
$$

We write $\varphi=\varphi^{+}-\varphi^{-}$and use 22 - 23 with $\varphi^{+}$and $\varphi^{-}$. Since $u$ is bounded and $\phi(u) \in$ $L^{2}(0, T ; V)$ we find that there exists a constant $C$ such as

$$
\forall \varphi \in \mathcal{D}(0, T ; V), \quad\left|\int_{0}^{T} \int_{\Omega_{p}} u \partial_{t} \varphi \mathrm{d} x \mathrm{~d} t\right| \leqslant C\|\varphi\|_{L^{2}(0, T ; V)},
$$

which ensures that $\partial_{t} u \in L^{2}\left(0, T ; V^{\prime}\right)$ (see Appendix of [3]). Thus,

$$
\forall \varphi \in \mathcal{D}(0, T ; V), \quad-\int_{0}^{T} \int_{\Omega_{p}} u \partial_{t} \varphi \mathrm{d} x \mathrm{~d} t=\int_{0}^{T}\left\langle\left\langle\partial_{t} u, \varphi\right\rangle\right\rangle \mathrm{d} t .
$$

Thus by density, we may rewrite (22) and (23) with $\varphi$ in $L^{2}(0, T ; V)$. Then we consider $\varphi=$ $(v-\phi(u))^{+}$and $\varphi=(v-\phi(u))^{-}$respectively, with $v$ as in the statement of Proposition 1 
so that, due to the obstacle condition for $u,(1+\operatorname{sgn}(u-\theta))(v-\phi(u))^{+}=0$ and respectively $(1-\operatorname{sgn}(u-\kappa))(v-\phi(u))^{-}=0$ a.e. on $Q_{p}$ and $\mathcal{H}^{n}$-a.e. on $\Sigma_{h p}$. By adding up, inequality 21] follows, which completes the proof of Proposition 1 .

\subsection{The uniqueness theorem}

Theorem 1 ensures a uniqueness property in the hyperbolic zone. In the parabolic one, the lack of regularity of the partial time derivative of a weak solution to (1)-(6) requires doubling the time variable and uses a suitable time-integration by parts formula. Furthermore, to deal with the convective terms, we assume that

$$
\zeta \mapsto K \circ \phi^{-1} \text { is Lipschitz continuous on } \phi\left(\mathcal{C}_{\theta}^{\infty}\right) .
$$

Then we have:

THEOREM 2 Under (24) problem (1)-6) admits at most one weak solution.

Proof. Let $u_{1}$ and $u_{2}$ be two weak solutions to (10-6). Thanks to Lemma 1 and Theorem 1 , we know that $u_{1}=u_{2}$ a.e. on $Q_{h}$. In addition, $\theta$ being independent of the time variable on $Q_{p}$ we may choose in [21], for $u_{1}$ written in variables $(t, x)$,

$$
v_{1}(t, x)=\phi\left(u_{1}\right)(t, x)-\frac{\mu \alpha_{\delta}}{\left\|\alpha_{\delta}\right\|_{\infty}} \operatorname{sgn}_{\mu}\left(\phi\left(u_{1}\right)(t, x)-\phi\left(u_{2}\right)(\tilde{t}, x)\right),
$$

and in 21) for $u_{2}$ written in variables $(\tilde{t}, x)$,

$$
v_{2}(\tilde{t}, x)=\phi\left(u_{2}\right)(\tilde{t}, x)+\frac{\mu \alpha_{\delta}}{\left\|\alpha_{\delta}\right\|_{\infty}} \operatorname{sgn}_{\mu}\left(\phi\left(u_{1}\right)(t, x)-\phi\left(u_{2}\right)(\tilde{t}, x)\right) .
$$

For any positive $\delta$, set

$$
\alpha_{\delta}(t, \tilde{t})=\gamma\left(\frac{t+\tilde{t}}{2}\right) \rho_{\delta}\left(\frac{t-\tilde{t}}{2}\right),
$$

where $\gamma \in \mathcal{D}(] 0, T[), \gamma \geqslant 0$, and $\delta$ is small enough for $\alpha_{\delta}$ to belong to $\mathcal{D}(] 0, T[\times] 0, T[)$. To simplify the writing we add a tilde to any function in the $\tilde{t}$ variable; $q$ stands for $(t, x)$ while $\tilde{q}$ stands for $(\tilde{t}, x)$. By adding up (and setting $\left.w_{\mu, \delta}\left(u_{1}, \tilde{u}_{2}\right)=\operatorname{sgn}_{\mu}\left(\phi\left(u_{1}\right)-\phi\left(\tilde{u}_{2}\right)\right) \alpha_{\delta}\right)$, we obtain

$$
\begin{aligned}
& \int_{] 0, T\left[\times Q_{p}\right.}\left\langle\left\langle\partial_{t} u_{1}-\partial_{\tilde{t}} u_{2}, w_{\mu, \delta}\left(u_{1}, \tilde{u}_{2}\right)\right\rangle\right\rangle \mathrm{d} t \mathrm{~d} \tilde{t}+\int_{] 0, T\left[\times Q_{p}\right.} \nabla\left\{\phi\left(u_{1}\right)-\phi\left(\tilde{u}_{2}\right)\right\} . \nabla w_{\mu, \delta}\left(u_{1}, \tilde{u}_{2}\right) \mathrm{d} q \mathrm{~d} \tilde{t} \\
& \leqslant-\int_{] 0, T\left[\times Q_{p}\right.}\left\{K\left(u_{1}\right)-K\left(\tilde{u}_{2}\right)\right\} \mathbf{B} \cdot \nabla w_{\mu, \delta}\left(u_{1}, \tilde{u}_{2}\right) \mathrm{d} q \mathrm{~d} \tilde{t} \\
&+\int_{] 0, T\left[\times Q_{p}\right.} K\left(\tilde{u}_{2}\right)(\mathbf{B}-\tilde{\mathbf{B}}) \cdot \nabla w_{\mu, \delta}\left(u_{1}, \tilde{u}_{2}\right) \mathrm{d} q \mathrm{~d} \tilde{t} \\
&-\int_{] 0, T\left[\times Q_{p}\right.}\left\{g_{p}\left(q, u_{1}\right)-g_{p}\left(\tilde{q}, \tilde{u}_{2}\right)\right\} w_{\mu, \delta}\left(u_{1}, \tilde{u}_{2}\right) \mathrm{d} q \mathrm{~d} \tilde{t} \\
&-\int_{0}^{T} \operatorname{ess}_{\tau \rightarrow 0^{-}} \int_{\Sigma_{h p}} K\left(u_{1}\left(\sigma+\tau v_{h}\right)\right) \mathbf{B} \cdot v_{h} w_{\mu, \delta}\left(u_{1}, \tilde{u}_{2}\right) \mathrm{d} \mathcal{H}_{\sigma}^{n} d \tilde{t} \\
&+\int_{0}^{T} \operatorname{ess}_{\tau \rightarrow 0^{-}} \int_{\Sigma_{h p}} K\left(u_{2}\left(\tilde{\sigma}+\tau v_{h}\right)\right) \tilde{\mathbf{B}}_{v_{h}} w_{\mu, \delta}\left(u_{1}, \tilde{u}_{2}\right) \mathrm{d} \mathcal{H}_{\tilde{\sigma}}^{n} \mathrm{~d} t .
\end{aligned}
$$


To deal with the first term on the left-hand side, we use a time-integration by parts formula in the same spirit as in [5, the Mignot-Bamberger lemma]. For the second integral on the righthand side, a Green formula is used since (24) ensures that $K\left(\tilde{u}_{2}\right)=\left(K \circ \phi^{-1}\right)\left(\phi\left(\tilde{u}_{2}\right)\right)$ belongs to $L^{2}\left(0, T ; H^{1}\left(\Omega_{p}\right)\right)$. For the boundary integrals we argue that due to uniqueness in the hyperbolic zone, $u_{2}\left(\tilde{\sigma}+\tau v_{h}\right)=u_{1}\left(\tilde{\sigma}+\tau v_{h}\right)$ for a.e. $(\tilde{\sigma}, \tau)$. This way, as a consequence of [20],

$$
\begin{aligned}
\int_{0}^{T} \underset{\tau \rightarrow 0^{-}}{\operatorname{ess} \lim } \int_{\Sigma_{h p}} K\left(u_{2}\left(\tilde{\sigma}+\tau v_{h}\right)\right) \tilde{\mathbf{B}} \cdot v_{h} w_{\mu, \delta}\left(u_{1}, \tilde{u}_{2}\right) \mathrm{d} \mathcal{H}_{\tilde{\sigma}}^{n} \mathrm{~d} t \\
=\int_{0}^{T} \underset{\tau \rightarrow 0^{-}}{\operatorname{ess} \lim } \int_{\Sigma_{h p}} K\left(u_{1}\left(\tilde{\sigma}+\tau v_{h}\right)\right) \tilde{\mathbf{B}} \cdot v_{h} w_{\mu, \delta}\left(u_{1}, \tilde{u}_{2}\right) \mathrm{d} \mathcal{H}_{\tilde{\sigma}}^{n} \mathrm{~d} t \\
=\int_{0}^{T} \int_{\Sigma_{h p}} \gamma(\tilde{\sigma}) w_{\mu, \delta}\left(u_{1}, \tilde{u}_{2}\right) \mathrm{d} \mathcal{H}_{\tilde{\sigma}}^{n} \mathrm{~d} t
\end{aligned}
$$

where $\gamma \in L^{\infty}\left(\Sigma_{h p}\right)$. It follows that

$$
\begin{aligned}
-\int_{] 0, T\left[\times Q_{p}\right.} & \left(\int_{\tilde{u}_{2}}^{u_{1}} \operatorname{sgn}_{\mu}\left(\phi(r)-\phi\left(\tilde{u}_{2}\right)\right) d r\right) \partial_{t} \alpha_{\delta} \mathrm{d} q \mathrm{~d} \tilde{t} \\
& -\int_{] 0, T\left[\times Q_{p}\right.}\left(\int_{\tilde{u}_{2}}^{u_{1}} \operatorname{sgn}_{\mu}\left(\phi\left(u_{1}\right)-\phi(r)\right) \mathrm{d} r\right) \partial_{\tilde{t}} \alpha_{\delta} \mathrm{d} q \mathrm{~d} \tilde{t} \\
\leqslant & \|\mathbf{B}\|_{L^{\infty}\left(Q_{p}\right)} \int_{] 0, T\left[\times Q_{p}\right.}\left|K\left(u_{1}\right)-K\left(\tilde{u}_{2}\right)\right|\left|\nabla w_{\mu, \delta}\left(u_{1}, \tilde{u}_{2}\right)\right|_{n} \mathrm{~d} q \mathrm{~d} \tilde{t} \\
& +\int_{] 0, T\left[\times Q_{p}\right.} \operatorname{div}\left(K\left(\tilde{u}_{2}\right)(\mathbf{B}-\tilde{\mathbf{B}})\right) w_{\mu, \delta}\left(u_{1}, \tilde{u}_{2}\right) \mathrm{d} q \mathrm{~d} \tilde{t} \\
& -\int_{] 0, T\left[\times \Sigma_{h p}\right.} K\left(\tilde{u}_{2}\right)(\mathbf{B}-\tilde{\mathbf{B}}) \cdot v_{h} w_{\mu, \delta}\left(u_{1}, \tilde{u}_{2}\right) \mathrm{d} q \mathrm{~d} \tilde{t} \\
& -\int_{] 0, T\left[\times Q_{p}\right.}\left\{g\left(q, u_{1}\right)-g\left(\tilde{q}, \tilde{u}_{2}\right)\right\} w_{\mu, \delta}\left(u_{1}, \tilde{u}_{2}\right) \mathrm{d} q \mathrm{~d} \tilde{t} \\
& -\int_{0}^{T} \int_{\Sigma_{h p}} \gamma(\sigma) w_{\mu, \delta}\left(u_{1}, \tilde{u}_{2}\right) \mathrm{d} \mathcal{H}_{\sigma}^{n} \mathrm{~d} \tilde{t}+\int_{0}^{T} \int_{\Sigma_{h p}} \gamma(\tilde{\sigma}) w_{\mu, \delta}\left(u_{1}, \tilde{u}_{2}\right) \mathrm{d} \mathcal{H}_{\tilde{\sigma}}^{n} \mathrm{~d} t .
\end{aligned}
$$

We let $\mu \rightarrow 0$. For the first integral on the right-hand side we refer to 24) and use the Saks lemma to deduce that it goes to 0 . Thus one has

$$
\begin{aligned}
& -\int_{] 0,1\left[\times Q_{p}\right.}\left|u_{1}-\tilde{u}_{2}\right|\left(\partial_{t} \alpha_{\delta}+\partial_{\tilde{t}} \alpha_{\delta}\right) \mathrm{d} q \mathrm{~d} \tilde{t} \\
& \leqslant \int_{] 0, T\left[\times Q_{p}\right.}\left|\operatorname{div}\left(K\left(\tilde{u}_{2}\right)(\mathbf{B}-\tilde{\mathbf{B}})\right)\right| \alpha_{\delta} \mathrm{d} q \mathrm{~d} \tilde{t}+\int_{] 0, T\left[\times \Sigma_{h p}\right.}\left|K\left(\tilde{u}_{2}\right)(\mathbf{B}-\tilde{\mathbf{B}}) \cdot v_{h}\right| \alpha_{\delta} \mathrm{d} q \mathrm{~d} \tilde{t} \\
& \quad+M_{g_{p}} \int_{] 0, T\left[\times Q_{p}\right.}\left|u_{1}-\tilde{u}_{2}\right| \alpha_{\delta} \mathrm{d} q \mathrm{~d} \tilde{t}+\int_{0}^{T} \int_{0}^{T} \int_{\Gamma_{h p}}|\gamma(t, s)-\gamma(\tilde{t}, s)| \alpha_{\delta} \mathrm{d} s \mathrm{~d} t \mathrm{~d} \tilde{t} .
\end{aligned}
$$

We return to the definition of $\alpha_{\delta}$ to express its partial derivatives with respect to $t$ and $\tilde{t}$. This way we may pass to the limit with $\delta$ through the classical argument of the Lebesgue points for an integrable 
function on $] 0, T[$ : all the terms on the right-hand side tend to 0 (B being smooth) except the first integral in the third line. The end is classical: it uses a piecewise linear approximation of $\mathbb{I}_{00, t[}$ for $t$ outside a set of measure zero. Thanks to [15] and to Gronwall's lemma we complete the proof of Theorem 2 .

\section{The existence property}

\subsection{The obstacle problem to the second order}

We intend to approximate the weak solution to (1)-(6) by a sequence of solutions to viscous problems deduced from (1)-6) by adding a diffusion term only in the hyperbolic area. This is in accordance with the proposed physical modelling of two layers in the subsoil with different geological characteristics. So for any positive $\epsilon$, we introduce

$$
\mathcal{T}_{\epsilon, h}(u)=\partial_{t} u-\sum_{i=1}^{n} \partial_{x_{i}}\left(\epsilon \partial_{x_{i}} \phi(u)+K(u) B_{i}\right)+g_{h}(t, x, u),
$$

and we consider the free boundary problem: find a measurable and bounded function $u_{\epsilon}$ on $Q$ such that formally (for the bilateral obstacle problem)

$$
\begin{array}{cccc}
0 \leqslant u_{\epsilon} \leqslant \theta & \text { on } Q, & \\
\mathcal{T}_{p}\left(u_{\epsilon}\right)=0 & \text { on } Q_{p} \cap\left[0<u_{\epsilon}<\theta\right], & \mathcal{T}_{\epsilon, h}\left(u_{\epsilon}\right)=0 & \text { on } Q_{h} \cap\left[0<u_{\epsilon}<\theta\right], \\
\mathcal{T}_{\epsilon, h}\left(u_{\epsilon}\right) \leqslant 0 & \text { on } Q_{h} \cap\left[0<u_{\epsilon}=\theta\right], & \mathcal{T}_{\epsilon, h}\left(u_{\epsilon}\right) \geqslant 0 & \text { on } Q_{h} \cap\left[0=u_{\epsilon}<\theta\right], \\
\mathcal{T}_{p}\left(u_{\epsilon}\right) \leqslant 0 & \text { on } Q_{p} \cap\left[0<u_{\epsilon}=\theta\right], & \mathcal{T}_{p}\left(u_{\epsilon}\right) \geqslant 0 & \text { on } Q_{p} \cap\left[0=u_{\epsilon}<\theta\right], \\
& u_{\epsilon}=0 \quad \text { on } \Sigma, \quad u_{\epsilon}(0, \cdot)=u_{0} & \text { on } \Omega,
\end{array}
$$

and to have a well-posed problem, we express the transmission conditions across the interface (which will be discussed in Remark 6)

$$
\begin{aligned}
-\epsilon \nabla \phi\left(u_{\epsilon}\right) \cdot v_{h}=\nabla \phi\left(u_{\epsilon}\right) \cdot v_{p} & \text { on } \Sigma_{h p} \cap\left[0<u_{\epsilon}<\theta\right], \\
u_{\epsilon \mid Q_{h}}=u_{\epsilon \mid Q_{p}} & \text { on } \Sigma_{h p} .
\end{aligned}
$$

Our aim is to prove first that 25] - 31] has a unique weak solution and secondly to establish some estimates suitable for the study of the behaviour of the sequence $\left(u_{\epsilon}\right)_{\epsilon>0}$ when $\epsilon$ goes to $0^{+}$. We obtain an existence result for (25)-(31) by using the artificial viscosity method-to regularize $\phi-$ and by relaxing the obstacle condition. That is why we start by introducing a Lipschitz bounded extension $K^{\star}$ and $g_{i}^{\star}$, for $i$ in $\{h, p\}$, of $K$ and $g_{i}$ outside $\mathcal{C}_{\theta}^{\infty}$ through (for a generic function $f$ )

$$
f^{\star}(z)= \begin{cases}f(z) & \text { if } z \in \mathcal{C}_{\theta}^{\infty}, \\ f\left(l_{\left.\mathcal{C}_{\theta}\right)}^{\infty}\right. & \text { if } z \leqslant l_{\mathcal{C}_{\theta}^{\infty},}^{\infty} \\ f\left(\operatorname{ess}_{\bar{\Omega}} \sup _{\bar{\Omega}}\right) & \text { if } z \geqslant \operatorname{ess} \sup _{\bar{\Omega}} \theta,\end{cases}
$$

where $l_{\mathcal{C}_{\theta}^{\infty}}=\min \mathcal{C}_{\theta}^{\infty}$ depending on the unilateral or bilateral case. For $\phi$ we choose an increasing Lipschitz extension $\phi^{\star}$ outside $\mathcal{C}_{\theta}^{\infty}$, so that due to [11), $\left(\phi^{\star}\right)^{-1}$ exists and is a continuous function on $\phi^{\star}\left(\mathcal{C}_{\theta}^{\infty}\right)$. 
Then, for any positive parameter $\eta$, we set $\phi_{\eta}^{\star}=\phi^{\star}+\eta \mathbb{I}_{\mathbb{R}}$ and $\beta(x, u)=-u^{-}+(u-\theta(x))^{+}$for a bilateral constraint (while for a unilateral obstacle condition $\beta$ is reduced to $\beta(x, u)=(u-\theta(x))^{+}$) and let $\lambda_{\epsilon, \eta}$ be a $\mathcal{C}^{1}(\bar{\Omega})$ approximation of $\lambda_{\epsilon}=\mathbb{I}_{\Omega_{p}}(x)+\epsilon \mathbb{I}_{\Omega_{h}}$ such that

$$
\begin{gathered}
\exists N>0, \forall \epsilon>0, \forall \eta>0, \quad 0<\lambda_{\epsilon, \eta} \leqslant N \text { a.e. in } \Omega, \\
\left\|\nabla \lambda_{\epsilon, \eta}\right\|_{\infty} \leqslant C(\epsilon) / \eta \quad \lambda_{\epsilon, \eta} \rightarrow \lambda_{\epsilon} \text { a.e. on } \Omega \text { as } \eta \rightarrow 0^{+} .
\end{gathered}
$$

This way we obtain (see e.g. [7])

THEOREM 3 There exists a unique solution

$$
u_{\epsilon, \eta} \in W\left(0, T ; H_{0}^{1}(\Omega) \cap H^{2}(\Omega) ; L^{2}(\Omega)\right) \cap L^{\infty}(Q)
$$

to the nondegenerate-penalized problem:

$$
\begin{aligned}
\partial_{t} u_{\epsilon, \eta}-\operatorname{div}\left(\lambda_{\epsilon, \eta}(x) \nabla \phi_{\eta}^{\star}\left(u_{\epsilon, \eta}\right)+K^{\star}\left(u_{\epsilon, \eta}\right) \mathbf{B}\right)+g^{\star}\left(t, x, u_{\epsilon, \eta}\right) & =-\frac{1}{\eta} \beta\left(x, u_{\epsilon, \eta}\right) \quad \text { a.e. on } Q \\
u_{\epsilon, \eta}(0, \cdot) & =u_{0} \quad \text { a.e. in } \Omega
\end{aligned}
$$

Now we state some a priori estimates for $\left(u_{\epsilon, \eta}\right)_{\eta>0}$ that are sufficient to study its limit when $\eta$ goes to $0^{+}$. Set $\left.Q_{s}=\right] 0, s[\times \Omega, s$ in $\left.] 0, T\right]$.

Proposition 2 There exists a constant $C$ independent of $\epsilon$ and $\eta$ such that:

$$
\begin{gathered}
\forall t \in[0, T], \quad M_{2}(t) \leqslant u_{\epsilon, \eta}(t, \cdot) \leqslant M_{1}(t) \quad \text { a.e. in } \Omega, \\
\left\|\beta\left(x, u_{\epsilon, \eta}\right)\right\|_{L^{1}(Q)} \leqslant C \eta, \\
\left\|\lambda_{\epsilon, \eta}^{1 / 2} \nabla \widehat{\phi_{\eta}^{\star}}\left(u_{\epsilon, \eta}\right)\right\|_{L^{2}(Q)^{n}} \leqslant C, \\
\forall s \in] 0, T], \quad \epsilon\left\|\partial_{t} \widehat{\phi_{\eta}^{\star}}\left(u_{\epsilon, \eta}\right)\right\|_{L^{2}\left(Q_{s}\right)}^{2}+\frac{\epsilon}{2}\left\|\lambda_{\epsilon, \eta}^{1 / 2} \nabla \phi_{\eta}^{\star}\left(u_{\epsilon, \eta}\right)(s, \cdot)\right\|_{L^{2}(\Omega)^{n}}^{2} \leqslant C,
\end{gathered}
$$

where $M_{1}$ and $M_{2}$ are defined in (9) and (10) and ${\widehat{\phi_{\eta}^{\star}}}_{\eta}(x)=\int_{0}^{x} \sqrt{\left(\phi^{\star}\right)_{\eta}^{\prime}(\tau)} \mathrm{d} \tau$.

Proof. For (34) we use a cut-off method in $L^{1}$ by considering the $L^{2}\left(Q_{s}\right)$-scalar product of (32) and $\operatorname{sgn}_{\mu}^{+}\left(u_{\epsilon, \eta}-M_{1}(t)\right)$ for the majorization by $M_{1}$, and $-\operatorname{sgn}_{\mu}^{-}\left(u_{\epsilon, \eta}-M_{2}(t)\right)$ for the minorization by $M_{2}$. A cut-off method in $L^{1}$ also provides $(35)$. From the energy equality satisfied by $u_{\epsilon, \eta}$ we derive (36). To conclude we take the $L^{2}\left(Q_{s}\right)$-scalar product of 32) and $\epsilon \partial_{t} \phi_{\eta}^{\star}\left(u_{\epsilon, \eta}\right)$. Concerning the penalized term, we have

$$
\begin{aligned}
\frac{\epsilon}{\eta} \int_{] 0, s[\times \Omega}\left(u_{\epsilon, \eta}-\theta\right)^{+} & \partial_{t} \phi_{\eta}^{\star}\left(u_{\epsilon, \eta}\right) \\
& =\frac{\epsilon}{\eta} \int_{] 0, s[\times \Omega}\left(u_{\epsilon, \eta}-\theta\right)^{+}\left(\phi_{\eta}^{\star}\right)^{\prime}\left(\left(u_{\epsilon, \eta}-\theta\right)^{+}+\theta\right) \partial_{t}\left(u_{\epsilon, \eta}-\theta\right)^{+} \mathrm{d} x \mathrm{~d} t \\
& =\frac{\epsilon}{\eta} \int_{] 0, s[\times \Omega} \partial_{t}\left(\int_{0}^{\left(u_{\epsilon, \eta}-\theta\right)^{+}} \tau\left(\phi_{\eta}^{\star}\right)^{\prime}(\tau+\theta) \mathrm{d} \tau\right) \mathrm{d} x \mathrm{~d} t \\
& =\frac{\epsilon}{\eta} \int_{\Omega}\left(\int_{0}^{\left(u_{\epsilon, \eta}(s, \cdot)-\theta\right)^{+}} \tau\left(\phi_{\eta}^{\star}\right)^{\prime}(\tau+\theta) \mathrm{d} \tau\right) \mathrm{d} x \geqslant 0 .
\end{aligned}
$$


The same reasoning and the same sign condition hold for $-\left(u_{\epsilon, \eta}\right)^{-}$. We bound the convective and reactive terms by using (34), (36) and the Young inequality (see [9]). Thanks to the density of $\mathcal{D}\left(0, T ; H_{0}^{1}(\Omega) \cap H^{2}(\Omega)\right)$ in $W\left(0, T ; H_{0}^{1}(\Omega) \cap H^{2}(\Omega) ; L^{2}(\Omega)\right)$, the diffusive term is integrated by parts and then with respect to $t$. Note that the constant $C$ in $(36)$ depends on $\left\|\phi\left(u_{0}\right)\right\|_{H_{0}^{1}(\Omega)}$ and $\left\|u_{0}\right\|_{H_{0}^{1}(\Omega)}$.

If the parameter $\epsilon$ is fixed, $\left(\phi_{\eta}^{\star}\left(u_{\epsilon, \eta}\right)\right)_{\eta>0}$ remains at least in a bounded subset of $H^{1}(Q)$. As a result, the compact embedding of the latter space into $L^{2}(Q)$ and the continuity of $\left(\phi^{\star}\right)^{-1}$ provide the existence of a measurable function $u_{\epsilon}$ and a subsequence - still denoted $\left(u_{\epsilon, \eta}\right)_{\eta>0}$ - such that when $\eta$ goes to $0^{+},\left(u_{\epsilon, \eta}\right)_{\eta>0}$ goes to $u_{\epsilon}$ in $L^{q}(Q), 1 \leqslant q<+\infty$, and $\left(\phi_{\eta}^{\star}\left(u_{\epsilon, \eta}\right)\right)_{\eta>0}$ goes to $\phi\left(u_{\epsilon}\right)$ weakly in $H^{1}(Q)$ and strongly in $\mathcal{C}^{0}\left([0, T] ; L^{2}(\Omega)\right)$. This leads to

THEOREM 4 Problem 25-31) has at least a weak solution $u_{\epsilon}$ such that

$$
\begin{gathered}
\forall t \in] 0, T\left[, \quad u_{\epsilon}(t, \cdot) \in \mathcal{C}_{\theta} \quad \text { a.e. in } \Omega,\right. \\
\phi\left(u_{\epsilon}\right) \in W\left(0, T ; H_{0}^{1}(\Omega) ; L^{2}(\Omega)\right), \\
u_{\epsilon}(0, \cdot)=u_{0} \quad \text { a.e. in } \Omega,
\end{gathered}
$$

and for any $v$ in $W\left(0, T ; H_{0}^{1}(\Omega) ; L^{2}(\Omega)\right), v(T, \cdot)=u_{\epsilon}(T, \cdot)$ a.e. in $\Omega$, such that for any $t$ in $[0, T]$, $\phi^{-1}(v(t, \cdot)) \in \mathcal{C}_{\theta}$ a.e. on $\Omega$,

$$
\begin{aligned}
\int_{Q} \partial_{t} v\left(v-\phi\left(u_{\epsilon}\right)\right) & \mathrm{d} x \mathrm{~d} t+\int_{Q} \lambda_{\epsilon}(x) \nabla \phi\left(u_{\epsilon}\right) \cdot \nabla\left(v-\phi\left(u_{\epsilon}\right)\right) \mathrm{d} x \mathrm{~d} t \\
& +\int_{Q} K\left(u_{\epsilon}\right) \mathbf{B} \cdot \nabla\left(v-\phi\left(u_{\epsilon}\right)\right) \mathrm{d} x \mathrm{~d} t+\int_{Q} g\left(t, x, u_{\epsilon}\right)\left(v-\phi\left(u_{\epsilon}\right)\right) \mathrm{d} x \mathrm{~d} t \\
& -\int_{Q}\left(u_{\epsilon}-v\right) \partial_{t}\left(v-\phi\left(u_{\epsilon}\right)\right) \mathrm{d} x \mathrm{~d} t \\
& +\int_{\Omega}\left(u_{0}-v(0, \cdot)\right)\left(\phi\left(u_{0}\right)-v(0, \cdot)\right) \mathrm{d} x \geqslant 0 .
\end{aligned}
$$

REMARK 4 In (38), (40), (41) the trace of $u_{\epsilon}$ with respect to the time variable has to be understood, for any $t$ in $[0, T]$, as $u_{\epsilon}(t, \cdot)=\phi^{-1}\left(\phi\left(u_{\epsilon}\right)(t, \cdot)\right)$.

Proof. The obstacle condition (38) follows from (35), while (40) comes from (33) and from the strong convergence of $\left(\phi_{\eta}^{\star}\left(u_{\epsilon, \eta}\right)\right)_{\eta>0}$ to $\phi\left(u_{\epsilon}\right)$ in $\mathcal{C}^{0}\left([0, T] ; L^{2}(\Omega)\right)$. To obtain 441 we take the $L^{2}(Q)$-scalar product of 32 and $v-\phi_{\eta}^{\star}\left(u_{\epsilon, \eta}\right)$. To study the penalized term, we write

$$
\begin{aligned}
-\frac{1}{\eta} \int_{Q}\left(u_{\epsilon, \eta}-\theta\right)^{+}\left(v-\phi_{\eta}^{\star}\left(u_{\epsilon, \eta}\right)\right) \mathrm{d} x \mathrm{~d} t= & -\frac{1}{\eta} \int_{Q}\left(u_{\epsilon, \eta}-\theta\right)^{+}\left(v-\phi^{\star}\left(u_{\epsilon, \eta}\right)\right) \mathrm{d} x \mathrm{~d} t \\
& +\int_{Q}\left(u_{\epsilon, \eta}-\theta\right)^{+} u_{\epsilon, \eta} \mathrm{d} x \mathrm{~d} t,
\end{aligned}
$$

where on the right-hand side the first term is nonnegative and the second one goes to 0 (due to (35)). The same reasoning is still true for the negative part of $\beta(x, \cdot)$. For the evolution term, we artificially introduce the quantity $\partial_{t} \phi(v)\left(\phi(v)-\phi^{\star}\left(u_{\epsilon, \eta}\right)\right)$. Then we integrate by parts in time and use the definition of $v$. This allows us to take the $\eta$-limit. Just note that in the diffusive term we take in fact the "lim inf" and apply the weak convergence of gradients in $L^{2}(Q)$. 
Now, we observe that

Proposition 3 If $u_{1}$ and $u_{2}$ are two weak solutions to (38)-41) for initial data $u_{0,1}$ and $u_{0,2}$ respectively, then (with $M_{g}=M_{g_{h}}+M_{g_{p}}$ ),

$$
\text { for a.e. } t \text { in }] 0, T\left[, \quad \int_{\Omega}\left|u_{1}(t, x)-u_{2}(t, x)\right| \mathrm{d} x \leqslant \int_{\Omega}\left|u_{0,1}-u_{0,2}\right| \mathrm{d} x e^{M_{g} t} .\right.
$$

Proof. We develop the same reasoning (on the whole $Q$ ) as in Theorem 2 (on $Q_{p}$ ) by doubling the time variable and using the same test functions (recall that $\theta$ is independent of the time variable on the whole $Q$ ). Observe that there are no boundary integrals here. Moreover, to deal with the evolution terms, we perform first an integration by parts with respect to the time variable assuming that $\alpha_{\delta}$ has a compact support in $] 0, T[\times] 0, T$ [. Then we apply the integration formula proved in [9] through some convexity inequalities:

LEMMA 3 Let $u$ be a bounded (by a constant $M$ ) measurable function on $Q$, and $f$ a function defined on $\Omega \times[-M, M]$ such that for any $x$ in $\Omega, \lambda \mapsto f(x, \lambda)$ is nondecreasing and continuous and for all $\lambda$ in $[-M, M], x \mapsto f(x, \lambda)$ is measurable and bounded on $\Omega$ and $\partial_{t} f(\cdot, u) \in L^{1}(Q)$. Then, for any $\alpha \in \mathcal{C}^{1}([0, T]), \alpha \geqslant 0$, such that $\alpha(T)=\alpha(0)=0$,

$$
\int_{Q} u \partial_{t}(f(x, u) \alpha) \mathrm{d} x \mathrm{~d} t=\int_{Q}\left(\int_{v}^{u} f(x, r) \mathrm{d} r\right) \partial_{t} \alpha \mathrm{d} x \mathrm{~d} t
$$

for any measurable function $v$ bounded by $M$ on $\Omega$.

This way,

$$
\begin{aligned}
\int_{] 0, T[\times Q} u_{1} \partial_{t}\left(\operatorname{sgn}_{\mu}\left(\phi\left(u_{1}\right)-\phi\left(\tilde{u}_{2}\right)\right) \alpha_{\delta}\right) \mathrm{d} q \mathrm{~d} \tilde{t} & -\int_{] 0, T[\times Q} \tilde{u}_{2} \partial_{\tilde{t}}\left(\operatorname{sgn}_{\mu}\left(\phi\left(u_{1}\right)-\phi\left(\tilde{u}_{2}\right)\right) \alpha_{\delta}\right) \mathrm{d} q \mathrm{~d} \tilde{t} \\
= & \int_{] 0, T[\times Q}\left(\int_{\tilde{u}_{2}}^{u_{1}} \operatorname{sgn}_{\mu}\left(\phi(r)-\phi\left(\tilde{u}_{2}\right)\right) d r\right) \partial_{t} \alpha_{\delta} \mathrm{d} q \mathrm{~d} \tilde{t} \\
& -\int_{] 0, T[\times Q}\left(\int_{u_{1}}^{\tilde{u}_{2}} \operatorname{sgn}_{\mu}\left(\phi\left(u_{1}\right)-\phi(r)\right) d r\right) \partial_{\tilde{t}} \alpha_{\delta} \mathrm{d} q \mathrm{~d} \tilde{t} .
\end{aligned}
$$

The conclusion follows.

\subsection{The viscous limit}

The uniqueness property stated in Proposition 3 ensures that the whole sequence $\left(u_{\epsilon, \eta}\right)_{\eta>0}$ converges to $u_{\epsilon}$ when $\eta$ goes to $0^{+}$. Thus, by considering the a priori estimates of Proposition 2 for $\left(u_{\epsilon, \eta}\right)_{\eta>0}$, we may derive some estimates for $\left(u_{\epsilon}\right)_{\epsilon>0}$. Indeed, we have

PROPOSITION 4

$$
\left(u_{\epsilon}\right)_{\epsilon>0} \text { is a bounded sequence in } L^{\infty}(Q),
$$

and there exists a constant $C$ independent of $\epsilon$ such that

$$
\epsilon^{1 / 2}\left\|\nabla \widehat{\phi}\left(u_{\epsilon}\right)\right\|_{L^{2}\left(Q_{h}\right)^{n}}+\left\|\nabla \widehat{\phi}\left(u_{\epsilon}\right)\right\|_{L^{2}\left(Q_{p}\right)^{n}} \leqslant C .
$$


Relations (42) and (43) are not sufficient to study the behaviour of the sequence $\left(u_{\epsilon}\right)_{\epsilon>0}$ when $\epsilon$ goes to $0^{+}$: we also need an estimate of $\partial_{t} u_{\epsilon}$ in a suitable space. For this purpose, we prove that $u_{\epsilon}$ satisfies an entropy inequality on $Q$ that will also be used as a starting point to establish (13) for the corresponding $\epsilon$-limit.

Proposition 5 Assume that

$$
\theta_{\mid \Omega_{h}} \text { belongs to } H^{2}\left(\Omega_{h}\right) .
$$

Then there exists a constant $C$ independent of $\epsilon$ such that

$$
\left\|\partial_{t} u_{\epsilon}\right\|_{L^{2}\left(0, T ; H^{-1}(\Omega)\right)} \leqslant C .
$$

Proof. We set $\kappa(x)=k \theta(x), k \in[0,1]$ for a bilateral constraint, and $\kappa(x)=k+\theta(x), M_{2}(T)-$ $\operatorname{ess} \sup _{\bar{\Omega}} \theta \leqslant k \leqslant 0$ for a unilateral obstacle condition. We consider the $L^{2}(Q)$-scalar product of (32) and $w_{\mu}^{\epsilon, \eta} \equiv \operatorname{sgn}_{\mu}\left(\phi_{\eta}^{\star}\left(u_{\epsilon, \eta}\right)-\phi_{\eta}(\kappa)\right) \zeta$, where $\zeta \in \mathcal{D}(]-\infty, T[\times \Omega), \zeta \geqslant 0$. We observe first that the penalized term is nonnegative. The other integrals are subjected to the following transformations: For the evolution term, with $I_{\mu}\left(u_{\epsilon, \eta}, \kappa\right)=\int_{\kappa}^{u_{\epsilon, \eta}} \operatorname{sgn}_{\mu}\left(\phi_{\eta}^{\star}(\tau)-\phi_{\eta}(\kappa)\right) \mathrm{d} \tau$,

$$
\begin{aligned}
\int_{Q} \partial_{t} u_{\epsilon, \eta} w_{\mu}^{\epsilon, \eta} \mathrm{d} x \mathrm{~d} t & =\int_{Q} \partial_{t} I_{\mu}\left(u_{\epsilon, \eta}, \kappa\right) \zeta \mathrm{d} x \mathrm{~d} t \\
& =-\int_{Q} I_{\mu}\left(u_{\epsilon, \eta}, \kappa\right) \partial_{t} \zeta \mathrm{d} x \mathrm{~d} t-\int_{\Omega} I_{\mu}\left(u_{0}, \kappa\right) \zeta(0, \cdot) \mathrm{d} x
\end{aligned}
$$

For the diffusion term,

$$
\begin{aligned}
\int_{Q} \lambda_{\epsilon, \eta} \nabla \phi_{\eta}^{\star}\left(u_{\epsilon, \eta}\right) . \nabla w_{\mu}^{\epsilon, \eta} \mathrm{d} x \mathrm{~d} t= & \int_{Q} \lambda_{\epsilon, \eta} \nabla\left(\phi_{\eta}^{\star}\left(u_{\epsilon, \eta}\right)-\phi_{\eta}(\kappa)\right) . \nabla w_{\mu}^{\epsilon, \eta} \mathrm{d} x \mathrm{~d} t \\
& +\int_{Q} \lambda_{\epsilon, \eta} \nabla \phi_{\eta}(\kappa) . \nabla w_{\mu}^{\epsilon, \eta} \mathrm{d} x \mathrm{~d} t .
\end{aligned}
$$

We develop the partial derivatives in the first term on the right-hand side and we use the fact that $\operatorname{sgn}_{\mu}($.$) is nondecreasing. To take the limit in \eta$, we recall that due to (34) and (37), $\left(w_{\mu}\left(u_{\epsilon, \eta}, \kappa\right)\right)_{\eta>0}$ is a bounded sequence in $H^{1}(Q) \cap L^{\infty}(Q)$, uniformly with respect to $\eta$ and so, thanks to the convergence properties of $\left(u_{\epsilon, \eta}\right)_{\eta>0}$ to $u_{\epsilon}$, converges to $w_{\mu}^{\epsilon} \equiv \operatorname{sgn}_{\mu}\left(\phi\left(u_{\epsilon}\right)-\phi(\kappa)\right) \zeta$ strongly in $L^{q}(Q), 1 \leqslant q<+\infty$, and weakly in $H^{1}(Q)$. Having taken the $\eta$-limit, we use the Green formula in the second diffusion term by dividing the integration range into $Q_{h}$ (where $\lambda_{\epsilon}=\epsilon$ ) and $Q_{p}$ (where $\lambda_{\epsilon}=1$ ). We obtain

$$
\begin{aligned}
-\int_{Q} I_{\mu}\left(u_{\epsilon}, \kappa\right) \partial_{t} \zeta \mathrm{d} x \mathrm{~d} t-\int_{\Omega} I_{\mu}\left(u_{0}, \kappa\right) \zeta(0, \cdot) \mathrm{d} x \\
+\int_{Q} \lambda_{\epsilon} \operatorname{sgn}_{\mu}\left(\phi\left(u_{\epsilon}\right)-\phi(\kappa)\right) \nabla\left(\phi\left(u_{\epsilon}\right)-\phi(\kappa)\right) . \nabla \zeta \mathrm{d} x \mathrm{~d} t \\
+\int_{Q}\left(K\left(u_{\epsilon}\right)-K(\kappa)\right) \mathbf{B} . \nabla w_{\mu}^{\epsilon} \mathrm{d} x \mathrm{~d} t+\int_{\Sigma_{h p}}(\epsilon-1) \nabla \phi(\kappa) \cdot \nu_{h} w_{\mu}^{\epsilon} \mathrm{d} \mathcal{H}^{n} \\
-\int_{Q}\left(\lambda_{\epsilon} \Delta \phi(\kappa)+\operatorname{div}(K(\kappa) \mathbf{B})-g\left(t, x, u_{\epsilon}\right)\right) w_{\mu}^{\epsilon} \mathrm{d} x \mathrm{~d} t \leqslant 0 .
\end{aligned}
$$


We let $\mu \rightarrow 0^{+}$using the Lebesgue dominated convergence theorem and the Saks lemma to deal with the first term in the third line (recall that 24) holds). It follows that

$$
\begin{aligned}
-\int_{Q} L\left(u_{\epsilon}, \kappa, \zeta\right) \mathrm{d} x \mathrm{~d} t-\int_{\Omega}\left|u_{0}-\kappa\right| \zeta(0, \cdot) \mathrm{d} x & \\
& +\int_{Q} \lambda_{\epsilon} \nabla\left|\phi\left(u_{\epsilon}\right)-\phi(\kappa)\right| . \nabla \zeta \mathrm{d} x \mathrm{~d} t-\int_{Q} \lambda_{\epsilon} \Delta \phi(\kappa) \operatorname{sgn}\left(u_{\epsilon}-\kappa\right) \zeta \mathrm{d} x \mathrm{~d} t \\
& +\int_{\Sigma_{h p}}(\epsilon-1) \nabla \phi(\kappa) \cdot v_{h} \operatorname{sgn}\left(\phi\left(u_{\epsilon}\right)-\phi(\kappa)\right) \zeta \mathrm{d} \mathcal{H}^{n} \leqslant 0 .
\end{aligned}
$$

Now the arguments are similar to those developed in Proposition 1 to prove that $\partial_{t} u$ is in $L^{2}\left(0, T ; V^{\prime}\right)$. In (47) we assume that $\zeta$ is a nonnegative element of $\mathcal{D}(Q)$ and so, thanks to a density argument, we may choose $\zeta$ in $\mathcal{D}\left(0, T ; H_{0}^{1}(\Omega)\right), \zeta \geqslant 0$. Thus for $k=1$ in the case of a bilateral obstacle and $k=0$ in the case of a unilateral one (so that $\kappa(x)=\theta(x)$ ), one has (with $\left.\mathcal{T}_{\epsilon}=\mathcal{T}_{\epsilon, h} \mathbb{I}_{\Omega_{h}}+\mathcal{T}_{p} \mathbb{I}_{\Omega_{p}}\right)$

$$
\begin{aligned}
\int_{Q} u_{\epsilon} \partial_{t} \zeta \mathrm{d} x \mathrm{~d} t \leqslant & \int_{Q} \lambda_{\epsilon} \nabla \phi\left(u_{\epsilon}\right) \cdot \nabla \zeta \mathrm{d} x \mathrm{~d} t+\int_{Q} K\left(u_{\epsilon}\right) \mathbf{B} \cdot \nabla \zeta \mathrm{d} x \mathrm{~d} t+\int_{Q} g\left(t, x, u_{\epsilon}\right) \zeta \mathrm{d} x \mathrm{~d} t \\
& -(\epsilon-1) \int_{\Sigma_{h p}} \nabla \phi(\theta) \cdot v_{h}\left(1+\operatorname{sgn}\left(\phi\left(u_{\epsilon}\right)-\phi(\theta)\right)\right) \zeta \mathrm{d} \mathcal{H}^{n} \\
& -\int_{Q}\left(1+\operatorname{sgn}\left(u_{\epsilon}-\theta\right)\right) \mathcal{T}_{\epsilon}(\theta) \zeta \mathrm{d} x \mathrm{~d} t
\end{aligned}
$$

and for $\kappa(x)=0$ in the case of a bilateral constraint and $\kappa(x)=M_{2}(T)-\operatorname{ess}_{\sup } \bar{\Omega} \theta \theta(x)$ for a unilateral one (thus $u_{\epsilon}-\kappa \geqslant 0$ a.e.) we have

$$
\begin{aligned}
\int_{Q} u_{\epsilon} \partial_{t} \zeta \mathrm{d} x \mathrm{~d} t \geqslant & \int_{Q} \lambda_{\epsilon} \nabla \phi\left(u_{\epsilon}\right) \cdot \nabla \zeta \mathrm{d} x \mathrm{~d} t+\int_{Q} K\left(x, u_{\epsilon}\right) \mathbf{B} \cdot \nabla \zeta \mathrm{d} x \mathrm{~d} t+\int_{Q} g\left(t, x, u_{\epsilon}\right) \zeta \mathrm{d} x \mathrm{~d} t \\
& -(\epsilon-1) \int_{\Sigma_{h p}} \nabla \phi(\kappa) \cdot \nu_{h}\left(1-\operatorname{sgn}\left(\phi\left(u_{\epsilon}\right)-\phi(\kappa)\right)\right) \zeta \mathrm{d} \mathcal{H}^{n} \\
& -\int_{Q}\left(1-\operatorname{sgn}\left(u_{\epsilon}-\kappa\right)\right) \mathcal{T}_{\epsilon}(\kappa) \zeta \mathrm{d} x \mathrm{~d} t .
\end{aligned}
$$

For any $\zeta$ in $\mathcal{D}\left(0, T ; H_{0}^{1}(\Omega)\right)$, we write $\zeta=\zeta^{+}-\zeta^{-}$and use the previous two inequalities with $\zeta^{+}$and $\zeta^{-}$. Thanks to the estimates of Proposition 4, and to the continuity of the trace operator from $V$ into $L^{2}\left(\Gamma_{h p}\right)$ (so from $H_{0}^{1}(\Omega)$ into $L^{2}\left(\Gamma_{h p}\right)$ ), we prove the existence of a constant $C$ (independent of $\epsilon$ ) such that

$$
\forall \zeta \in \mathcal{D}\left(0, T ; H_{0}^{1}(\Omega)\right), \quad\left|\int_{Q} u_{\epsilon} \partial_{t} \zeta \mathrm{d} x \mathrm{~d} t\right| \leqslant C\|\zeta\|_{L^{2}\left(0, T ; H_{0}^{1}(\Omega)\right)} .
$$

Thus $\partial_{t} u_{\epsilon} \in L^{2}\left(0, T ; H^{-1}(\Omega)\right)$ and for any $\zeta \in \mathcal{D}\left(0, T ; H_{0}^{1}(\Omega)\right)$,

$$
-\int_{Q} u_{\epsilon} \partial_{t} \zeta \mathrm{d} x \mathrm{~d} t=\int_{0}^{T}\left\langle\partial_{t} u_{\epsilon}, \zeta\right\rangle \mathrm{d} t .
$$

Thus estimate (45) follows, which completes the proof of Proposition 5 . 
REMARK 5 By applying [9], we may assert that as soon as $\partial_{t} u_{\epsilon} \in L^{2}\left(0, T ; H^{-1}(\Omega)\right)$ we can perform a time-integration by parts in (41) so that the weak solution to (25)-(29) satisfies the "strong" variational inequality for any measurable function $v \in \mathcal{C}_{\theta}$ a.e. on $\Omega$ with $\phi(v)$ in $H_{0}^{1}(\Omega)$,

$$
\begin{aligned}
\left\langle\partial_{t} u_{\epsilon}, \phi(v)-\phi\left(u_{\epsilon}\right)\right\rangle+\int_{\Omega} & \left(\lambda_{\epsilon} \nabla \phi\left(u_{\epsilon}\right)+K\left(u_{\epsilon}\right) \mathbf{B}\right) \cdot \nabla\left(\phi(v)-\phi\left(u_{\epsilon}\right)\right) \mathrm{d} x \\
& \left.+\int_{\Omega} g\left(t, x, u_{\epsilon}\right)\left(\phi(v)-\phi\left(u_{\epsilon}\right)\right) \mathrm{d} x \geqslant 0 \quad \text { for a.e. } t \text { in }\right] 0, T[.
\end{aligned}
$$

REMARK 6 By reasoning as in Remark 3 and setting $\mathbf{V}_{\epsilon}=\left(u_{\epsilon},-\lambda_{\epsilon} \nabla \phi\left(u_{\epsilon}\right)-K\left(u_{\epsilon}\right) \mathbf{B}\right)$ we show that $\mathbf{V}_{\epsilon \mid Q_{i}} \in H_{\operatorname{div}_{(t, x)}}\left(Q_{i}\right)$ and a.e. on $Q_{i}$,

$$
\begin{aligned}
& -\operatorname{div}_{(t, x)} \mathbf{V}_{\epsilon \mid Q_{i}} \leqslant g_{i}\left(t, x, u_{\epsilon}\right)-\left(1+\operatorname{sgn}\left(u_{\epsilon}-\theta\right)\right) \mathcal{T}_{i}(\theta), \\
& -\operatorname{div}_{(t, x)} \mathbf{V}_{\epsilon \mid Q_{i}} \geqslant g_{i}\left(t, x, u_{\epsilon}\right)-\left(1-\operatorname{sgn}\left(u_{\epsilon}-\kappa\right)\right) \mathcal{T}_{i}(\kappa) .
\end{aligned}
$$

Therefore if $\left[0<u_{\epsilon}<\theta\right]$ is an open subset of $Q, \mathcal{H}^{n}$-measurable, we obtain

$$
\operatorname{div}_{(t, x)}\left(\mathbf{V}_{\epsilon, \mid Q_{i}}\right)=0 \quad \text { on } Q_{i} \cap\left[0<u_{\epsilon}<\theta\right],
$$

which is 26), and for any nonnegative $\zeta$ with support in $Q \cap\left[0<u_{\epsilon}<\theta\right]$,

$$
\left\lfloor\mathbf{V}_{\epsilon \mid Q_{h}} \cdot v_{h}+\mathbf{V}_{\epsilon \mid Q_{p}} \cdot v_{p}, \zeta\right\rfloor=0,
$$

which corresponds to 30]. Observe that [31) holds since $\phi\left(u_{\epsilon}\right) \in H^{1}(Q)$. Finally, $\nabla \phi(\theta) . v_{h} \geqslant$ $\mathcal{T}_{\epsilon}(\theta) \mathcal{H}^{n}$-a.e. on $\Sigma_{h p} \cap\left[0<u_{\epsilon}=\theta\right]$.

To study the behaviour of the sequence $\left(u_{\epsilon}\right)_{\epsilon>0}$ and characterize the corresponding limit we need an additional assumption on $\phi$ :

$$
\left.\phi^{-1} \text { is Hölder continuous on } \phi\left(\mathcal{C}_{\theta}^{\infty}\right) \text { with an exponent } \tau \text { in }\right] 0,1[.
$$

We then have

Proposition 6 If $(48)$ holds, then there exists a measurable function $u$ in $L^{\infty}(Q)$, with $u(t, \cdot) \in$ $\mathcal{C}_{\theta}$ a.e. on $\Omega$ for a.e. $t$ in $] 0, T\left[, \phi(u)\right.$ in $L^{2}(0, T ; V)$ and such that up to a subsequence, when $\epsilon$ goes to $0^{+}$,

$$
\begin{aligned}
& u_{\epsilon} \rightarrow u \text { in } L^{\infty}(Q) \text { weak- } \star \text {, and in } L^{q}\left(Q_{p}\right), 1 \leqslant q<+\infty, \\
& \nabla \phi\left(u_{\epsilon}\right) \rightarrow \nabla \phi(u) \quad \text { weakly in } L^{2}\left(Q_{p}\right)^{n}, \quad \epsilon \nabla \phi\left(u_{\epsilon}\right) \rightarrow 0^{+} \quad \text { strongly in } L^{2}\left(Q_{h}\right)^{n} .
\end{aligned}
$$

Proof. The strong convergence in $L^{q}\left(Q_{p}\right)$ for $\left(u_{\epsilon}\right)_{\epsilon>0}$ refers to the arguments put forward in [5. Chapter 2]. From (45) the sequence $\left(\partial_{t} u_{\epsilon}\right)_{\epsilon>0}$ remains fixed in a bounded subset of $L^{2}\left(0, T ; H^{-1}\left(\Omega_{p}\right)\right)$ and due to 43 , the sequence $\left(\phi\left(u_{\epsilon}\right)\right)_{\epsilon>0}$ is bounded in $L^{2}(0, T ; V)$ uniformly with respect to $\epsilon$. Using the fact that

$$
\forall s \in] 0,1\left[, \quad L^{2}(0, T ; V) \hookrightarrow L^{2}\left(0, T ; H^{1}\left(\Omega_{p}\right)\right) \hookrightarrow L^{2}\left(0, T ; W^{s, 2}\left(\Omega_{p}\right)\right),\right.
$$

we infer that $u_{\epsilon} \equiv \phi^{-1}\left(\phi\left(u_{\epsilon}\right)\right)$ is bounded in $L^{2 / \tau}\left(0, T ; W^{\tau s, 2 / \tau}\left(\Omega_{p}\right)\right)$. The compact embedding of $W^{\tau s, 2 / \tau}\left(\Omega_{p}\right)$ into $L^{2 / \tau}\left(\Omega_{p}\right)$ and the J.-L. Lions compactness theorem ([10, p. 57]) ensure that $\mathcal{W} \equiv\left\{v \in L^{2 / \tau}\left(0, T ; W^{\tau s, 2 / \tau}\left(\Omega_{p}\right)\right): \partial_{t} v \in L^{2}\left(0, T ; H^{-1}\left(\Omega_{p}\right)\right)\right\}$ is compactly embedded in $L^{2 / \tau}\left(0, T ; L^{2 / \tau}\left(\Omega_{p}\right)\right)$. 
The above convergence properties for $\left(u_{\epsilon}\right)_{\epsilon>0}$ are sufficient to characterize the function $u$. In the hyperbolic zone we take advantage of (42) and of:

Claim 1 (see [4]) If $\mathcal{O}$ is an open bounded subset of $\mathbb{R}^{q}(q \geqslant 1)$ and $\left(u_{n}\right)_{n>0}$ a sequence of measurable functions on $\mathcal{O}$ such that

$$
\exists M>0, \forall n>0, \quad\left\|u_{n}\right\|_{L^{\infty}(\mathcal{O})} \leqslant M,
$$

then there exist a subsequence $\left(u_{\varphi(n)}\right)_{n>0}$ and a measurable $\pi$ in $L^{\infty}(] 0,1[\times \mathcal{O})$ such that for all bounded continuous functions $f$ on $\mathcal{O} \times]-M, M[$,

$$
\forall \xi \in L^{1}(\mathcal{O}), \quad \lim _{n \rightarrow+\infty} \int_{\mathcal{O}} f\left(x, u_{\varphi(n)}\right) \xi \mathrm{d} x=\int_{] 0,1[\times \mathcal{O}} f(x, \pi(\alpha, w)) \mathrm{d} \alpha \xi \mathrm{d} x .
$$

Such a result has first been applied to the approximation through the artificial viscosity method of the Cauchy problem in $\mathbb{R}^{p}$ for conservation laws, as one can establish a uniform $L^{\infty}$-control of approximate solutions. It has also been applied to the numerical analysis of transport equations since "finite volume" schemes only give an $L^{\infty}$-estimate uniformly with respect to the mesh length of the numerical solution (see [4]). Here the approximating sequence is a sequence of solutions to viscous problems 25]-29] and we state:

THEOREM 5 If

$$
\left(K \circ \phi^{-1}\right)^{\prime} \text { is continuous on } \phi\left(\mathcal{C}_{\theta}^{\infty}\right),
$$

then (1)- 6 has a weak solution that is the limit in $L^{q}(Q), 1 \leqslant q<+\infty$, of the whole sequence of solutions to viscous problems (25)-(31) when $\epsilon$ goes to $0^{+}$.

Proof. Let $u$ be as in Proposition 6 Since $\left(u_{\epsilon \mid \Omega_{h}}\right)_{\epsilon>0}$ is uniformly bounded, there exist a subsequence-still labelled $\left(u_{\epsilon \mid \Omega_{h}}\right)_{\epsilon>0}$ - and a bounded measurable function $\pi$-called a processon ]0,1[ $\times Q_{h}$ such that for any bounded continuous function $\psi$ on $Q_{h} \times \mathcal{C}_{\theta}^{\infty}$ and for any $\xi$ in $L^{1}\left(Q_{h}\right)$,

$$
\lim _{\epsilon \rightarrow 0^{+}} \int_{Q_{h}} \psi\left(t, x, u_{\epsilon}\right) \xi \mathrm{d} x \mathrm{~d} t=\int_{] 0,1\left[\times Q_{h}\right.} \psi(t, x, \pi(\alpha, t, x)) \xi \mathrm{d} \alpha \mathrm{d} x \mathrm{~d} t .
$$

We first establish that on $Q_{h}$, the process $\pi$ is reduced to $u_{\mid \Omega_{h}}$ and secondly we prove that $u$ is a weak solution to (1)-(6) for initial data $u_{0}$. To do so, we return to (46) in order to take the $\epsilon$-limit and then the $\mu$-limit separately in the parabolic and in the hyperbolic zone. Thanks to the convergence properties of $\left(u_{\epsilon}\right)_{\epsilon>0}$ there are no difficulties passing to these limits in the integrals over $Q_{p}$. In particular, for the convective term we refer to the Saks lemma. For the boundary integrals we use the fact that $\left(\operatorname{sgn}_{\mu}\left(\phi\left(u_{\epsilon}\right)-\phi(\kappa)\right) \zeta\right)_{\epsilon>0}$ is a bounded sequence in $L^{2}(0, T ; V)$ that weakly converges to $\operatorname{sgn}_{\mu}(\phi(u)-\phi(\kappa)) \zeta$ in $L^{2}(0, T ; V)$ up to a subsequence. Then we invoke the continuity and linearity of the trace operator from $V$ into $L^{2}\left(\Gamma_{h p}\right)$. In the hyperbolic zone, we take the $\epsilon$-limit thanks to (50) since all the nonlinearities are continuous with respect to $u_{\epsilon}$. However, the flux term

$$
I_{\epsilon, \mu}=\int_{Q_{h}} K\left(u_{\epsilon}\right) \operatorname{sgn}_{\mu}^{\prime}\left(\phi\left(u_{\epsilon}\right)-\phi(\kappa)\right) \nabla\left(\phi\left(u_{\epsilon}\right)-\phi(\kappa)\right) . \mathbf{B} \zeta \mathrm{d} x \mathrm{~d} t
$$

has to be carefully studied since we only have weak convergence of $\left(u_{\epsilon}\right)_{\epsilon>0}$ and of $\left(\nabla \phi\left(u_{\epsilon}\right)\right)_{\epsilon>0}$. That is why we introduce

$$
H_{\mu}(v, w)=\int_{w}^{v}\left(K \circ \phi^{-1}\right)(\tau) \operatorname{sgn}_{\mu}^{\prime}(\tau-w) \mathrm{d} \tau
$$


After an integration by parts with respect to $\tau$, we obtain

$$
\begin{aligned}
I_{\epsilon, \mu}= & \int_{Q_{h}} \nabla\left(H_{\mu}\left(\phi\left(u_{\epsilon}\right), \phi(\kappa)\right)\right) \cdot \mathbf{B} \zeta \mathrm{d} x \mathrm{~d} t \\
& -\int_{Q_{h}}\left(\int_{\phi(\kappa)}^{\phi\left(u_{\epsilon}\right)}\left(K \circ \phi^{-1}\right)^{\prime}(\tau) \operatorname{sgn}_{\mu}^{\prime}(\tau-\phi(\kappa)) \mathrm{d} \tau\right) \nabla \phi(\kappa) . \mathbf{B} \zeta \mathrm{d} x \mathrm{~d} t .
\end{aligned}
$$

Thanks to the Green formula,

$$
\begin{aligned}
I_{\epsilon, \mu}= & -\int_{Q_{h}} H_{\mu}\left(\phi\left(u_{\epsilon}\right), \phi(\kappa)\right)(\zeta \operatorname{div} \mathbf{B}+\nabla \zeta . \mathbf{B}) \mathrm{d} x \mathrm{~d} t+\int_{\Sigma_{h p}} H_{\mu}\left(\phi\left(u_{\epsilon}\right), \phi(\kappa)\right) \mathbf{B} \cdot v_{h} \zeta \mathrm{d} \mathcal{H}^{n} \\
& -\int_{Q_{h}}\left(\int_{\phi(\kappa)}^{\phi\left(u_{\epsilon}\right)}\left(K \circ \phi^{-1}\right)^{\prime}(\tau) \operatorname{sgn}_{\mu}^{\prime}(\tau-\phi(\kappa)) \mathrm{d} \tau\right) \nabla \phi(\kappa) . \mathbf{B} \zeta \mathrm{d} x \mathrm{~d} t .
\end{aligned}
$$

Since $\phi\left(u_{\epsilon}\right) \in L^{2}\left(0, T ; H^{1}(\Omega)\right)$, for a.e. $t$ in $] 0, T\left[,\left(\phi\left(u_{\epsilon}\right)_{\mid \Omega_{h}}\right)_{\mid \Gamma_{h p}}=\left(\phi\left(u_{\epsilon}\right)_{\mid \Omega_{p}}\right)_{\mid \Gamma_{h p}}\right.$. We now take the $\epsilon$-limit using (50). For the boundary integral, we argue as previously by noting that $\left(H_{\mu}\left(\phi\left(u_{\epsilon}\right), \phi(\kappa)\right) \zeta\right)_{\epsilon>0}$ is a bounded sequence in $L^{2}(0, T ; V)$. We get $\lim _{\epsilon \rightarrow 0^{+}} I_{\epsilon, \mu}=I_{\mu}$ where

$$
\begin{aligned}
I_{\mu}= & -\int_{\left.Q_{h} \times\right] 0,1[} H_{\mu}(\phi(\pi), \phi(\kappa))(\zeta \operatorname{div} \mathbf{B}+\nabla \zeta . \mathbf{B}) \mathrm{d} \alpha \mathrm{d} x \mathrm{~d} t+\int_{\Sigma_{h p}} H_{\mu}(\phi(u), \phi(\kappa)) \mathbf{B} \cdot v_{h} \zeta \mathrm{d} \mathcal{H}^{n} \\
& -\int_{\left.Q_{h} \times\right] 0,1[}\left(\int_{\phi(\kappa)}^{\phi(\pi)}\left(K \circ \phi^{-1}\right)^{\prime}(\tau) \operatorname{sgn}_{\mu}^{\prime}(\tau-\phi(\kappa)) \mathrm{d} \tau\right) \nabla \phi(\kappa) . \mathbf{B} \zeta \mathrm{d} \alpha \mathrm{d} x \mathrm{~d} t .
\end{aligned}
$$

To let $\mu \rightarrow 0^{+}$, we recall the definition of $\operatorname{sign}_{\mu}^{\prime}$ and we use the fact that since $K \circ \phi^{-1}$ is continuous on $\phi\left(\mathcal{C}_{\theta}^{\infty}\right),\left(H_{\mu}(v, w)\right)_{\mu>0}$ converges to $\operatorname{sgn}(v-w) K(w)$ a.e. on $\left.Q_{h} \times\right] 0,1\left[\right.$ and $\mathcal{H}^{n}$-a.e. on $\Sigma_{h p}$. In the same way, $\left(\int_{w}^{v}\left(K \circ \phi^{-1}\right)^{\prime}(\tau) \operatorname{sgn}_{\mu}^{\prime}(\tau-w) \mathrm{d} \tau\right)_{\mu>0}$ converges to $\operatorname{sgn}(v-w)\left(K \circ \phi^{-1}\right)^{\prime}(w)$ a.e. on $\left.Q_{h} \times\right] 0,1[$, by (49]. From the Lebesgue dominated convergence theorem, it follows that $\lim _{\mu \rightarrow 0^{+}} I_{\mu}=I$ where

$$
\begin{aligned}
I= & -\int_{\left.Q_{h} \times\right] 0,1[} \operatorname{sgn}(\pi-\kappa)(K(\kappa) \mathbf{B} \cdot \nabla \zeta+\zeta \operatorname{div}(K(\kappa) \mathbf{B})) \mathrm{d} \alpha \mathrm{d} x \mathrm{~d} t \\
& +\int_{\Sigma_{h p}} \operatorname{sgn}(\phi(u)-\phi(\kappa)) K(\kappa) \mathbf{B} \cdot v_{h} \zeta \mathrm{d} \mathcal{H}^{n},
\end{aligned}
$$

since $\phi$ is strictly increasing. Finally,

$$
\begin{aligned}
-\int_{Q_{p}} L_{p}(u, \kappa, \zeta) \mathrm{d} x & \mathrm{~d} t-\int_{\left.Q_{h} \times\right] 0,1[} L_{h}(\pi, \kappa, \zeta) \mathrm{d} \alpha \mathrm{d} x \mathrm{~d} t-\int_{\Omega}\left|u_{0}-\kappa\right| \zeta(0, \cdot) \mathrm{d} x \\
& +\int_{Q_{p}} \nabla|\phi(u)-\phi(\kappa)| . \nabla \zeta \mathrm{d} x \mathrm{~d} t-\int_{Q_{p}} \Delta \phi(\kappa) \operatorname{sgn}(u-\kappa) \zeta \mathrm{d} x \mathrm{~d} t \\
& -\int_{\Sigma_{h p}} \nabla \phi(\kappa) \cdot v_{h} \operatorname{sgn}(\phi(u)-\phi(\kappa)) \zeta \mathrm{d} \mathcal{H}^{n} \leqslant 0 .
\end{aligned}
$$


For $\zeta$ in $\left.\mathcal{D}(]-\infty, T] \times \Omega_{h}\right)$, we deduce that

$$
-\int_{\left.Q_{h} \times\right] 0,1[} L_{h}(\pi, \kappa, \zeta) \mathrm{d} \alpha \mathrm{d} x \mathrm{~d} t \leqslant \int_{\Omega_{h}}\left|u_{0}-\kappa\right| \zeta(0, \cdot) \mathrm{d} x .
$$

Therefore, following F. Otto's ideas in [11], but here in the context of a process solution, we established that

$$
\underset{t \rightarrow 0^{+}}{\operatorname{ess} \lim } \int_{] 0,1\left[\times \Omega_{h}\right.}|\pi(\alpha, t, x)-\Lambda(x)| \mathrm{d} \alpha \mathrm{d} x \leqslant \int_{\Omega_{h}}\left|u_{0}-\Lambda(x)\right| \mathrm{d} x,
$$

where $\Lambda(x)=k(x) \theta(x), k(\cdot)$ being a measurable function on $\Omega_{h}, 0 \leqslant k \leqslant 1$ a.e. in $\Omega_{h}$ for the bilateral obstacle problem and $\Lambda(x)=k(x)+\theta(x), M_{2}(T)-\operatorname{ess}_{\sup } \bar{\Omega} \theta \leqslant \leqslant 0$ a.e. in $\Omega_{h}$ for the unilateral one. The initial condition (15) on $\Omega_{h}$ for $\pi$ is obtained by choosing

$$
\begin{aligned}
& \Lambda(x)=\left\{\begin{array}{ll}
\frac{u_{0}(x)}{\theta(x)} & \text { if } \theta(x) \neq 0, \\
0 & \text { else },
\end{array}\right. \text { for the bilateral constraint, } \\
& \Lambda(x)=u_{0}(x)-\theta(x) \quad \text { for the unilateral one. }
\end{aligned}
$$

Now to establish (14) for $\pi$, we take advantage of the approximation properties of $u$ through $\left(u_{\epsilon}\right)_{\epsilon>0}$ and of $u_{\epsilon}$ through $\left(u_{\epsilon, \eta}\right)_{\eta>0}$ to return to (32)-(33) and consider the $L^{2}(Q)$-scalar product (32) and $\partial_{1} H_{l}\left(u_{\epsilon, \eta}, \kappa\right) \zeta$, where $\zeta \in \mathcal{D}(] 0, T\left[\times \bar{\Omega}_{h}\right), \zeta \geqslant 0, \zeta(t, \cdot)=0$ on $\Gamma_{h p}$ for any $t$ in [0,T], and

$$
\begin{aligned}
\forall l \in \mathbb{N}^{\star}, \quad H_{l}(z, w) & =\left((\operatorname{dist}(z,[0, w]))^{2}+\left(\frac{1}{l}\right)^{2}\right)^{1 / 2}-\frac{1}{l}, \\
\mathcal{Q}_{l}(z, w) & =\int_{w}^{z} \partial_{1} H_{l}(\tau, w)\left(K^{\star}\right)^{\prime}(\tau) \mathrm{d} \tau,
\end{aligned}
$$

is the family of boundary entropy-entropy flux pairs introduced by F. Otto [11]. We emphasize that $\partial_{1} H_{l}\left(u_{\epsilon, \eta}, \kappa\right) \zeta \in W\left(0, T ; H_{0}^{1}\left(\Omega_{h}\right) ; L^{2}\left(\Omega_{h}\right)\right)$ so that calculations may be performed as if we were in a single domain. In particular, the Green formula does not give rise to integrals along the interface. Since $0 \leqslant \partial_{1} H_{l}\left(u_{\epsilon, \eta}, \kappa\right) \beta\left(x, u_{\epsilon, \eta}\right)$ a.e. on $Q_{h}$,

$$
\begin{aligned}
-\int_{Q_{h}}\left(H_{l}\left(u_{\epsilon, \eta}, \kappa\right)\right. & \left.\partial_{t} \zeta-\mathcal{Q}_{l}\left(u_{\epsilon, \eta}, \kappa\right) \mathbf{B} \cdot \nabla \zeta-G_{h, l}\left(u_{\epsilon, \eta}, \kappa\right) \zeta\right) \mathrm{d} x \mathrm{~d} t \\
& \leqslant-\int_{Q_{h}} \lambda_{\epsilon, \eta}\left(\partial_{1} H_{l}\left(u_{\epsilon, \eta}, \kappa\right) \nabla \zeta+\zeta \partial_{21}^{2} H_{l}\left(u_{\epsilon, \eta}, \kappa\right) \nabla \kappa\right) . \nabla \phi_{\eta}^{\star}\left(u_{\epsilon, \eta}\right) \mathrm{d} x \mathrm{~d} t,
\end{aligned}
$$

taking into account the convexity of the function $\xi \mapsto H_{l}(\xi, \cdot)$ and

$$
\begin{aligned}
G_{h, l}\left(u_{\epsilon, \eta}, \kappa\right)= & \int_{\kappa}^{u_{\epsilon, \eta}}\left(\left(K^{\star}\right)^{\prime}(\tau) \mathbf{B} \cdot \nabla \kappa+K^{\star}(\tau) \operatorname{div} \mathbf{B}\right) \partial_{11}^{2} H_{l}(\tau, \kappa) \mathrm{d} \tau \\
& +g_{h}^{\star}\left(t, x, u_{\epsilon, \eta}\right) \partial_{1} H_{l}\left(u_{\epsilon, \eta}, \kappa\right) .
\end{aligned}
$$

On account of the convergence properties of $\left(u_{\epsilon, \eta}\right)_{\eta>0}$, we take the $\eta$-limit. Then, as previously, we take the $\epsilon$-limit thanks to 50 . It follows that

$$
-\int_{] 0,1\left[\times Q_{h}\right.}\left(H_{l}(\pi, \kappa) \partial_{t} \zeta-\mathcal{Q}_{l}(\pi, \kappa) \mathbf{B} \cdot \nabla \zeta-G_{h, l}(\pi, \kappa) \zeta\right) \mathrm{d} \alpha \mathrm{d} x \mathrm{~d} t \leqslant 0 .
$$


At this point, we adapt F. Otto's work, which yields

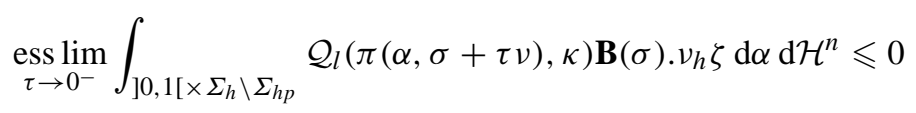

for any $\zeta$ in $L^{1}\left(\Sigma_{h} \backslash \Sigma_{h p}\right), \zeta \geqslant 0$. The boundary condition 14 for $\pi$ follows by observing that $\left(\mathcal{Q}_{l}\right)_{l \in \mathbb{N}^{*}}$ converges uniformly to $\mathcal{F}(z, 0, \kappa)$ as $l \rightarrow+\infty$.

So $\pi$ satisfies (14), 15] and (18) with similar integration ranges. This way, by reasoning as in Theorem 1 if $\pi_{1}(\alpha, \cdot, \cdot)$ and $\pi_{2}(\beta, \cdot, \cdot)$ are two process solutions for initial data $u_{0,1}$ and $u_{0,2}$, then for a.e. $t$ in $] 0, T[$,

$$
\int_{] 0,1\left[\times \Omega_{h}\right.}\left|\pi_{1}(\alpha, t, x)-\pi_{2}(\beta, t, x)\right| \mathrm{d} \alpha \mathrm{d} \beta \mathrm{d} x \mathrm{~d} t \leqslant \int_{\Omega_{h}}\left|u_{0,1}-u_{0,2}\right| \mathrm{d} x e^{M_{g_{h}} t} .
$$

When $u_{0,1}=u_{0,2}$ on $\Omega_{h}$, there exists a measurable function $u_{h}$ on $Q_{h}$ such that a.e. on $Q_{h}$, $u_{h}=\pi_{1}(\alpha, \cdot)=\pi_{2}(\beta, \cdot)$ for a.e. $\alpha$ and $\beta$ in ]0, 1 [. Moreover, the uniqueness property warrants that the whole sequence $\left(u_{\epsilon}\right)_{\epsilon>0}$ strongly converges to $u_{h}$ in $L^{q}\left(Q_{h}\right), 1 \leqslant q<+\infty$. Thus $u_{h}=u_{\mid \Omega_{h}}$ a.e. on $Q_{h}$ and $u$ satisfies (12)-(14). To complete the proof of Theorem 5 we only need to check that $u$ satisfies (15). Owing to (52) we just have to concentrate on $\Omega_{p}$. We consider (51) for $\zeta(t, x)=$ $\psi(t) \zeta(x)$ with $\psi$ in $\mathcal{D}(]-\infty, T[), \psi \geqslant 0$, and $\zeta$ in $\mathcal{D}\left(\Omega_{p}\right), \zeta \geqslant 0$ :

$$
-\int_{0}^{T}\left(\int_{\Omega_{p}}|u-k| \zeta \mathrm{d} x+f(t)\right) \psi^{\prime}(t) \mathrm{d} t \leqslant \int_{\Omega_{p}}\left|u_{0}-k\right| \zeta \psi(0) \mathrm{d} x,
$$

with

$$
\begin{aligned}
f(t)= & \int_{\Omega_{p}}\left(\int_{0}^{t}[-|K(u(\tau, x))-K(\kappa)| \mathbf{B} \cdot \nabla \zeta\right. \\
& \left.\left.+g_{p}(\tau, x, u(\tau, x)) \operatorname{sgn}(u(\tau, x)-k) \zeta-|\phi(u(\tau, x))-\phi(\kappa)| \Delta \zeta\right] \mathrm{d} \tau\right) \mathrm{d} x
\end{aligned}
$$

Hence the function $t \mapsto \int_{\Omega_{p}}|u-\kappa| \zeta \mathrm{d} x+f(t)$ is identified a.e. with a bounded nonincreasing function, so it has an essential limit as $t$ goes to $0^{+}, t$ in $] 0, T[\backslash \mathcal{O}$, where $\mathcal{L}(\mathcal{O})=0$. As $f$ goes to 0 with $t$, it follows that

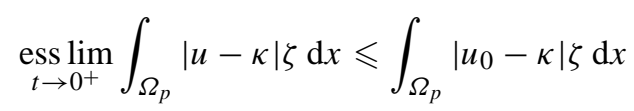

for any $\zeta \in \mathcal{D}\left(\Omega_{p}\right), \zeta \geqslant 0$. As a consequence, thanks to F. Otto's reasoning in [11] we obtain (with $\Lambda$ as in (52) )

$$
\underset{t \rightarrow 0^{+}}{\operatorname{ess} \lim } \int_{\Omega_{p}}|u(t, x)-\Lambda(x)| \mathrm{d} x \leqslant \int_{\Omega_{p}}\left|u_{0}-\Lambda(x)\right| \mathrm{d} x
$$

and we argue as for (52), which concludes the proof of Theorem 5 .

\section{REFERENCES}

1. Aguilar, G., Lévi, L., \& Madaune-Tort, M. Coupling of multidimensional parabolic and hyperbolic equations. J. Hyperbolic Differ. Equ. 3 (2006), 53-80. Zbl 1096.35092 MR 2214162 
2. Bia, P., \& Combarnous, M. Les méthodes thermiques de production des hydrocarbures. Rev. Inst. Français du Pétrole 1975, 359-394.

3. BRÉZIS, H. Opérateurs maximaux monotones et semi-groupes de contractions dans les espaces de Hilbert. North-Holland Math. Stud. 5, North-Holland, Amsterdam (1973). Zbl 0252.47055 MR 0348562

4. Eymard, R., Gallouët, T., \& Herbin, R. Existence and uniqueness of the entropy solution to a nonlinear hyperbolic equation. Chin. Ann. Math. Ser. B 16 (1995), 1-14. Zbl 0830.35077 MR 1338923

5. Gagneux, G., \& Madaune-Tort, M. Analyse mathématique de modèles non linéaires de l'ingénierie pétrolière. Math. Appl. (Paris) 22, Springer, Paris (1995). Zbl 0842.35126 MR 1616513

6. KobAyASI, K. The equivalence of weak solutions and entropy solutions of nonlinear degenerate secondorder equations. J. Differential Equations 189 (2003), 383-395. Zbl 1027.35058 MR 1964471

7. Ladyzhenskaya, O. A., Solonnikov, V. A., \& URal'tseva, N. N. Linear and Quasi-linear Equations of Parabolic Type. Transl. Math. Monogr. 23, Amer. Math. Soc., Providence, RI (1968). Zbl 0174.15403 MR 0241821

8. LÉvi, L. Obstacle problems for scalar conservation laws. M2AN Math. Model. Numer. Anal. 35 (2001), 575-593. Zbl 0990.35096 MR 1837085

9. LÉVI, L. The singular limit of a bilateral obstacle problem for a class of degenerate parabolic-hyperbolic operators. Adv. Appl. Math. 35 (2005), 34-57. Zbl 1074.35010 MR 2141504

10. Lions, J.-L. Quelques méthodes de résolution des problèmes aux limites non linéaires. Dunod and Gauthier-Villars, Paris (1969). Zbl 0189.40603 MR 0259693

11. Отто, F. Initial-boundary value problem for a scalar conservation law, C. R. Acad. Sci. Paris Sér. I 322 (1996), 729-734. Zbl 0852.35013 MR 1387428

12. Peyroutet, F., \& Madaune-Tort, M. Error estimate for a splitting method applied to convectionreaction equations, Math. Models Appl. Sci. 11 (2001), 1081-1100. Zbl 1013.35051 MR 1850564 